\title{
Midpoints and Exact Points of Some Algebraic Functions in Floating-Point Arithmetic
}

\author{
Claude-Pierre Jeannerod, Member, IEEE, Nicolas Louvet, \\ Jean-Michel Muller, Senior Member, IEEE, and Adrien Panhaleux
}

\begin{abstract}
When implementing a function $f$ in floating-point arithmetic, if we wish correct rounding and good performance, it is important to know if there are input floating-point values $x$ such that $f(x)$ is either the middle of two consecutive floating-point numbers (assuming rounded-to-nearest arithmetic), or a floating-point number (assuming rounded toward $\pm \infty$ or toward 0 arithmetic). In the first case, we say that $f(x)$ is a midpoint, and in the second case, we say that $f(x)$ is an exact point. For some usual algebraic functions and various floating-point formats, we prove whether or not there exist midpoints or exact points. When there exist midpoints or exact points, we characterize them or list all of them (if there are not too many). The results and the techniques presented in this paper can be used in particular to deal with both the binary and the decimal formats defined in the IEEE 754-2008 standard for floating-point arithmetic.
\end{abstract}

Index Terms-Floating-point arithmetic, correct rounding, algebraic function.

\section{INTRODUCTION}

$\mathrm{I}$ $\mathrm{N}$ a floating-point system that follows the IEEE 754-1985 standard for radix-2 floating-point arithmetic [1], the user can choose an active rounding mode, also called roundingdirection attribute in the newly revised IEEE 754-2008 standard [5]: rounding toward $-\infty$, rounding toward $+\infty$, rounding toward 0 , and rounding to nearest, which is the default rounding mode. Given a real number $x$, we denote, respectively, by $\mathrm{RD}(x), \mathrm{RU}(x), \mathrm{RZ}(x)$, and $\mathrm{RN}(x)$ these rounding modes. Let us also recall that correct rounding is required by the above cited IEEE standards for the four elementary arithmetic operations $(+,-, \times$, and $\div)$ as well as for the square root: the result of an operation is said to be correctly rounded if for any inputs, its result is the infinitely precise result rounded according to the active rounding mode. We are interested here in facilitating the delivery of correctly rounded results for various simple algebraic functions that are frequently used in numerical analysis or signal processing.

Let us call midpoint for a floating-point format a number that is exactly halfway between two consecutive floatingpoint numbers of that format. Given a function $f: \mathbb{R}^{d} \rightarrow \mathbb{R}$

- C.-P. Jeannerod is with INRIA Rhône-Alpes, LIP (UMR 5668 CNRS-ENS de Lyon-INRIA-UCBL), Université de Lyon, 46 allée d'Italie, F69364 Lyon Cedex 07, France.

E-mail: claude-pierre.jeannerod@ens-lyon.fr.

- N. Louvet is with UCBL Lyon 1, LIP (UMR 5668 CNRS-ENS de Lyon-INRIA-UCBL), Université de Lyon, 46 allée d'Italie, F69364 Lyon Cedex 07, France. E-mail: nicolas.louvet@ens-lyon.fr.

- J.-M. Muller is with CNRS, LIP (UMR 5668 CNRS-ENS de Lyon-INRIA-UCBL), Université de Lyon, 46 allée d'Italie, F69364 Lyon Cedex 07, France. E-mail: jean-michel.muller@ens-lyon.fr.

- A. Panhaleux is with ENS Lyon-Université de Lyon, LIP (UMR 5668 CNRS-ENS de Lyon-INRIA-UCBL), 46 allée d'Italie, F69364 Lyon Cedex 07, France. E-mail: adrien.panhaleux@ens-lyon.fr.

Manuscript received 7 Aug. 2009; revised 24 Mar. 2010; accepted 19 Apr. 2010; published online 11 June 2010.

Recommended for acceptance by J. Bruguera, M. Cornea, and D. Das Sarma. For information on obtaining reprints of this article, please send e-mail to: tc@computer.org, and reference IEEECS Log Number TCSI-2009-08-0374.

Digital Object Identifier no. 10.1109/TC.2010.144. and a floating-point vector $x$, we say that $f(x)$ is a midpoint of $f$ if $f(x)$ is a midpoint for that format.

Given $f$ and $x$, the problem of computing $\operatorname{RN}(f(x))$ is closely related to the knowledge of the midpoints of the function $f$. Then, a common strategy (see [15] and [10, chapter 10]) for returning $\mathrm{RN}(f(x))$ is as follows:

Let us first compute an approximation $f_{1}$, of accuracy $\epsilon_{1}$, to $f(x)$. If there are no midpoints of the considered floatingpoint format within distance $\epsilon_{1}$ from $f_{1}$, then necessarily $\mathrm{RN}(f(x))=\mathrm{RN}\left(f_{1}\right)$. If on the contrary, there are such midpoints within distance $\epsilon_{1}$ from $f_{1}$, we can progressively increase the quality of the approximations (that is, computing an approximation $f_{2}$ of accuracy $\epsilon_{2}<\epsilon_{1}$, and so on) until we are able to provide a correctly rounded result. The point is that this strategy may not terminate if the function $f$ has midpoints. As a consequence, a correctly rounded implementation of a given function $f$ can be made more efficient if we know in advance that $f$ admits no midpoints. If $f$ admits midpoints, it is also very useful to know how to characterize them.

If now we consider one of the directed rounding modes (RD, $\mathrm{RU}$, or RZ), the strategy that consists in progressively refining the approximations will not terminate if $f(x)$ is a floating-point number. In this case, we say that $f(x)$ is an exact point of the function $f$, and it is also very useful to know a characterization of these exact points when implementing $f$. Moreover, a characterization of the exact points of $f$ can be used to set the "inexact" flag required by the IEEE standards [1], [5]. For example, for $x / \sqrt{x^{2}+y^{2}}$ in radix 2, our study shows that this flag must always be raised except when $x$ or $y$ is zero, which can be detected easily.

In this paper, we present results on the existence of midpoints and exact points for some algebraic functions: beyond division, inversion, and square root, we study functions like the reciprocal square root $1 / \sqrt{y}$, the $2 \mathrm{D}$ euclidean norm $\sqrt{x^{2}+y^{2}}$ and its reciprocal $1 / \sqrt{x^{2}+y^{2}}$, 


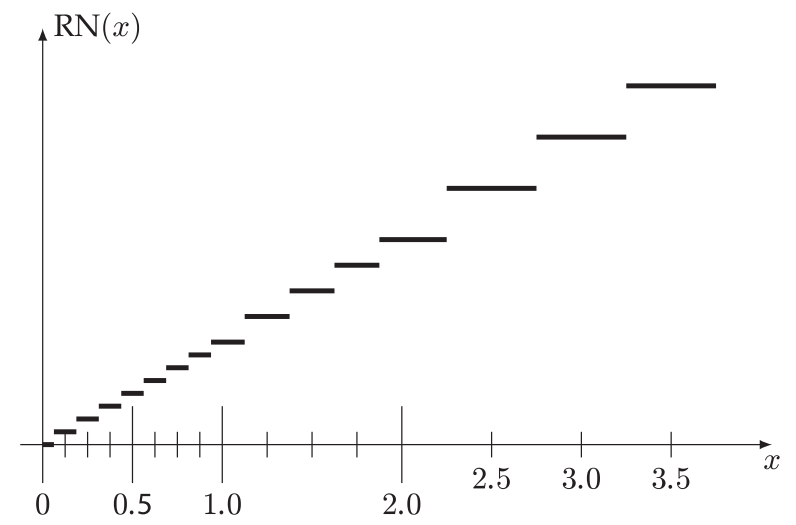

Fig. 1. The $\mathrm{RN}(x)$ function (radix $\beta=2$ and precision $p=3$ ).

and the 2D-normalization function $x / \sqrt{x^{2}+y^{2}}$. A part of the results presented on division and square root have been known for some time in binary arithmetic; see, for instance, the pioneering work by Markstein [9], as well as studies by Iordache and Matula [6] and Parks [11]. Let us also recall the work by Lauter and Lefèvre [8] on the function $x^{y}$, which thus covers integer powers. We present these results for completeness, and extend some of them to other radices, in particular to radix 10 .

Before going into further details, we introduce some definitions. A radix- $\beta$, precision- $p$ floating-point number $x$ is either 0 or a rational number of the form

$$
x= \pm X \cdot \beta^{e_{x}-p+1},
$$

where $X$ is a positive integer such that $X<\beta^{p}$. If in addition $\beta^{p-1} \leq X$, then $x= \pm X \cdot \beta^{e_{x}-p+1}$ is called the normalized representation of $x$, and the integers $X$ and $e_{x}$ are called, respectively, the integral significand and the exponent of $x$. We can, in fact, speak of the exponent for any nonzero real $x$ : in radix $\beta$, it is the unique integer $e_{x}$ such that $\beta^{e_{x}} \leq|x|<\beta^{e_{x}+1}$. On computing systems conforming to the IEEE $754-2008$ standard [5], the radix $\beta$ is 2 or 10 . Radix 16 is also sometimes used [12]. The exponent $e_{x}$ is bounded: $e_{\min } \leq e_{x} \leq e_{\max }$, where $e_{\min }$ and $e_{\max }$ are the extremal exponents of the considered floating-point format. A nonzero number without a normal representation is said subnormal: all subnormal numbers have absolute value less than $\beta^{e_{\text {min }}}$.

Assuming that we are working with a radix- $\beta$, precision- $p$ floating-point arithmetic, a midpoint is a rational number of the form

$$
z= \pm(Z+1 / 2) \cdot \beta^{e_{z}-p+1}
$$

where $Z$ is a nonnegative integer such that

$$
\begin{cases}\beta^{p-1} \leq Z<\beta^{p}, & \text { if } e_{\min }<e_{z} \leq e_{\max } \\ 0 \leq Z<\beta^{p}, & \text { if } e_{z}=e_{\min } .\end{cases}
$$

Such a number is exactly halfway between two consecutive floating-point numbers. The midpoints are the values where the function $x \mapsto \mathrm{RN}(x)$ is discontinuous, as illustrated in Fig. 1 on a toy floating-point format $\left(\beta=2, p=3, e_{\min }=-1\right.$, and $e_{\max }=1$ ).
TABLE 1

Summary of the Results Given in This Paper

\begin{tabular}{|c||c|c|c|c|}
\hline \multicolumn{1}{|c||}{} & \multicolumn{2}{c|}{ Midpoints } & \multicolumn{2}{c|}{ Exact points } \\
\hline $\begin{array}{c}\text { Function } \\
\sqrt{y}\end{array}$ & $\begin{array}{c}\text { Radix 2 } \\
\text { none }\end{array}$ & $\begin{array}{c}\text { Radix 10 } \\
\text { none }\end{array}$ & $\begin{array}{c}\text { Radix 2 } \\
\text { many }\end{array}$ & $\begin{array}{c}\text { Radix 10 } \\
\text { many }\end{array}$ \\
\hline $\begin{array}{c}1 / \sqrt{y} \\
x^{k} \text { for } \\
k \in \mathbb{N}_{>0}\end{array}$ & none & Theorem 3 & $y=2^{2 k}$ & Theorem 5 \\
\hline$x /\|y\|_{2}$ & none & many & many & many \\
\hline$x / y$ & none & many & many & many \\
\hline $1 / y$ & none & Theorem 8 & $y= \pm 2^{k}$ & Theorem 9 \\
\hline$\frac{1}{\sqrt{x^{2}+y^{2}}}$ & none & Theorem 12 & $\begin{array}{c}\{x, y\}= \\
\left\{0, \pm 2^{k}\right\}\end{array}$ & Theorem 13 \\
\hline$\frac{x}{\sqrt{x^{2}+y^{2}}}$ & none & none & $\begin{array}{c}x=0 \\
\text { or } y=0\end{array}$ & many \\
\hline$\sqrt{x^{2}+y^{2}}$ & many & many & many & many \\
\hline
\end{tabular}

When using the implementation of a mathematical function in floating-point arithmetic, in most practical cases, the input and output precisions are the same. However, a user may, for example, wish to calculate the singleprecision/binary32 number that is closest to the square root of a double-precision/binary64 floating-point number. For the sake of simplicity, we assume, in this paper, that the input and output precisions are the same. Moreover, we give our results assuming an unbounded exponent range, that is, under the hypothesis that no underflow nor overflow occurs. For that purpose, we define $\mathbb{F}_{\beta, p}$ as the set of the radix $-\beta$, precision- $p$ floating-point numbers, with an unbounded exponent range. Similarly, midpoints are restricted to the set

$$
\begin{aligned}
\mathbf{M I}_{\beta, p}= & \left\{ \pm(Z+1 / 2) \cdot \beta^{e_{z}-p+1}:\right. \\
& \left.Z \in \mathbb{N}, \beta^{p-1} \leq Z<\beta^{p}, e_{z} \in \mathbb{Z}\right\},
\end{aligned}
$$

where $\mathbb{Z}$ denotes the set of integers and $\mathbb{N}$ denotes the set $\{0,1,2, \ldots\}$ of nonnegative integers.

The purpose of this paper is, for the floating-point number systems and the algebraic functions mentioned above, to investigate whether these functions admit midpoints or exact points, and to characterize such midpoints and exact points when they exist. The results we obtain are for $\beta=2^{q}$ with $q$ a positive integer, and for $\beta=10$, but in some cases, we managed to weaken these assumptions on $\beta$. Moreover, most of the examples proposed are based on the basic formats defined in the IEEE 754-2008 standard [5] that are briefly recalled below:

\begin{tabular}{|l|c||l|c|}
\hline Binary formats & $p$ & Decimal formats & $p$ \\
\hline \hline binary32 & 24 & decimal32 & 7 \\
binary64 & 53 & decimal64 & 16 \\
binary128 & 113 & decimal128 & 34 \\
\hline
\end{tabular}

Table 1 summarizes the results presented in the paper. In this table, "many" indicates that the techniques we used did not allow us to find a simple characterization of the midpoints or of the exact points of the function, an exhaustive enumeration was impractical because of the too large number of cases to consider, and we have experimental evidence that the number of midpoints and/or exact points is 
large. Most of the results displayed here for $\beta=2$ are, in fact, obtained in a more general setting, namely, for $\beta=2^{q}$, where $q$ is a positive integer.

Note that since we considered an unbounded exponent range, subnormal floating-point numbers of the various IEEE 754-2008 formats can be written in normalized form. Hence, subnormal numbers are a subset of floating-point numbers with unbounded exponent range. This implies that the results presented in Table 1 remain unchanged when the inputs are subnormal numbers. If there are no exact points or midpoints for normal floating-point numbers with unbounded exponent range for a given function, then midpoints or exact points cannot occur if the inputs are subnormals. Similarly, if the exact points and midpoints are characterized by one of the theorems, assuming that the inputs are subnormals will only restrict the characterization of the theorem, without creating new possible exact points or midpoints.

However, some results presented in Table 1 change when we want to know if a given function outputs midpoints in the range of subnormal floating-point numbers. In radix 2, division admits midpoints in the subnormal range, as well as the function $x /\|y\|_{2}$, while they have no midpoints in the normal range. The square root function admits no midpoints, even in the subnormal range, for the square root of a floatingpoint number cannot be in the subnormal range. Although the results are not detailed in the paper, the techniques presented can be used to deal with midpoints in the subnormal range for the other functions listed in Table 1.

Outline. We start with extensions to radices $2^{q}$ and 10 of classical, radix-2 results for square roots (Section 2), reciprocal square roots (Section 3), and positive integer powers (Section 4). In Section 5, we move to the function that maps a real $x$ and a $d$-dimensional real vector $y=\left[y_{k}\right]_{1 \leq k \leq d}$ to $x /\|y\|_{2}$. Here, $\|\cdot\|_{2}$ denotes the euclidean norm of vectors:

$$
\|y\|_{2}=\sqrt{y_{1}^{2}+\cdots+y_{d}^{2}}
$$

The function $x /\|y\|_{2}$ is interesting for it covers several important special cases, each of them being detailed in a subsequent section: for $d=1$, division and reciprocal (Sections 6 and 7); for $d=2$, reciprocal 2D euclidean norm $1 / \sqrt{x^{2}+y^{2}}$ and normalization of $2 \mathrm{D}$ vectors $x / \sqrt{x^{2}+y^{2}}$ (Sections 8 and 9). We comment on the 2D euclidean norm in Section 10.

Notation. Throughout the paper, the symbols $\mathbb{Q}, \mathbb{R}$, and $\mathbb{N}_{>0}$ denote the rational numbers, the real numbers, and the positive integers, respectively. We write $i$ for the complex number whose square is -1 , and $\lfloor\cdot\rfloor$ and $\lceil\cdot\rceil$ for the usual floor and ceiling functions. Also, for $x, y \in \mathbb{Z}$ such that $y \neq 0$, we use the standard notation $x \bmod y=x-y\lfloor x / y\rfloor$ (see, for instance, Graham et al. [4, p. 82]).

\section{Square Root}

\subsection{Midpoints for Square Root}

The following theorem can be viewed as a consequence of a result of Markstein [9, Theorem 9.4]. It says that the square root function has no midpoints, whatever the radix $\beta$ is. A detailed proof is given here for completeness.
Theorem 1 (Markstein [9]). Let $y \in \mathbb{F}_{\beta, p}$ be positive. Then, $\sqrt{y} \notin \mathbb{M I}_{\beta, p}$.

Proof. Let $z=\sqrt{y}$ and assume that $z$ is in $\mathbb{M I}_{\beta, p}$. Then, there exist some integers $Z$ and $e_{z}$ such that $z=(Z+1 / 2)$. $\beta^{e_{z}-p+1}$ and $\beta^{p-1} \leq Z<\beta^{p}$. Using $y=z^{2}$ and $y=$ $Y \cdot \beta^{e_{y}-p+1}$, we deduce that

$$
4 Y \cdot \beta^{e_{y}-2 e_{z}+p-1}=(2 Z+1)^{2} .
$$

Now, one may check that $e_{z}=\left\lfloor e_{y} / 2\right\rfloor$ so that

$$
e_{y}-2 e_{z}=e_{y} \bmod 2,
$$

which is nonnegative. Thus, for $p \geq 1$, the left-hand side of (1) is an even integer. This contradicts the fact that the right-hand side is an odd integer.

\subsection{Exact Points for Square Root}

We saw in the previous section that the square root function has no midpoints. The situation for exact points is just opposite: for a given input exponent, the number $N$ of floating-point numbers having this exponent and whose square root is also a floating-point number grows essentially like $\beta^{p / 2}$. In this section, we make this claim precise for $\beta=2^{q}\left(q \in \mathbb{N}_{>0}\right)$ and $\beta=10$ by giving an explicit expression for $N$ in Theorem 2. To establish this counting formula, we need the following two lemmas:

Lemma 1. For $a, b \in \mathbb{R}$ such that $0 \leq a \leq b$, and $c \in \mathbb{N}_{>0}$, the number of integer multiples of $c$ that lie in $[a, b)$ is $\lceil b / c\rceil-\lceil a / c\rceil$.

Proof. Let us write $N_{a, b}^{(c)}$ for the number of integer multiples of $c$ lying in $[a, b)$. Since $0 \leq a \leq b$, the set $[0, b)$ is the union of the disjoint sets $[0, a)$ and $[a, b)$. Hence, $N_{a, b}^{(c)}=N_{0, b}^{(c)}-N_{0, a}^{(c)}$ and it remains to check that $N_{0, a}^{(c)}=\lceil a / c\rceil$. If $a \notin \mathbb{N}$, it follows from $c \in \mathbb{N}_{>0}$ that $N_{0, a}^{(c)}=1+\lfloor a / c\rfloor$. If $a \in \mathbb{N}$, either $c$ divides $a$ in which case $N_{0, a}^{(c)}=a / c$; otherwise, $N_{0, a}^{(c)}=1+\lfloor a / c\rfloor$.

Lemma 2. Let $y \in \mathbb{F}_{\beta, p}$ be positive. The real number $\sqrt{y}$ is also in $\mathbb{F}_{\beta, p}$ if and only if the integral significand $Y$ of $y$ satisfies $\beta^{p-1} \leq Y<\beta^{p}$ and $Y=Z^{2} \cdot \beta^{1-p-\left(e_{y} \bmod 2\right)}$ for some integer $Z$ such that $\beta^{p-1} \leq Z<\beta^{p}$.

Proof. Let $z=\sqrt{y}$. Assume first that $z \in \mathbb{F}_{\beta, p}$. Then, there exists an integer $Z$ such that $z=Z \cdot \beta^{e_{z}-p+1}$ and $\beta^{p-1} \leq$ $Z<\beta^{p}$. Using $y=z^{2}$ and $y=Y \cdot \beta^{e_{y}-p+1}$, we deduce that

$$
Y=Z^{2} \cdot \beta^{1-p-\left(e_{y}-2 e_{z}\right)} .
$$

The "only if" statement then follows from (2). Conversely, using $y=Y \cdot \beta^{e_{y}-p+1}$, we may rewrite the equality $Y=Z^{2} \cdot \beta^{1-p-\left(e_{y} \bmod 2\right)}$ as

$$
\sqrt{y}=Z \cdot \beta^{e_{z}-p+1},
$$

where

$$
e_{z}=\left(e_{y}-\left(e_{y} \bmod 2\right)\right) / 2 .
$$

By definition, $e_{z}$ is an integer and by assumption, $Z$ is an integer lying in $\left[\beta^{p-1}, \beta^{p}\right)$. Hence, $\sqrt{y}$ is in $\mathbb{F}_{\beta, p}$. 
Theorem 2. For a given exponent $e_{y}$, let $N$ denote the number of positive values $y \in \mathbb{F}_{\beta, p}$ such that $\sqrt{y} \in \mathbb{F}_{\beta, p}$, and let $\epsilon_{y}=\left(e_{y}+p-1\right) \bmod 2$.

- If $\beta=2^{q}, q \in \mathbb{N}_{>0}$, then

$$
N=\left\lceil 2^{\left(q p-\epsilon_{y}(q \bmod 2)\right) / 2}\right\rceil-\left\lceil 2^{\left(q(p-1)-\epsilon_{y}(q \bmod 2)\right) / 2}\right\rceil .
$$

- If $\beta=10$, then $N=\left\lceil 10^{\left(p-\epsilon_{y}\right) / 2}\right\rceil-\left\lceil 10^{\left(p-1-\epsilon_{y}\right) / 2}\right\rceil$.

Proof. Let $\gamma=p-1+\left(e_{y} \bmod 2\right)$. From Lemma $2, N$ is the number of integers $Y$ in $\left[\beta^{p-1}, \beta^{p}\right)$ and of the form $Z^{2}$. $\beta^{-\gamma}$ for some integer $Z$ such that $\beta^{p-1} \leq Z<\beta^{p}$.

Rewriting $Y=Z^{2} \cdot \beta^{-\gamma}$ as $Y \cdot \beta^{\epsilon_{y}} \cdot \beta^{\gamma-\epsilon_{y}}=Z^{2}$, we see that $\beta^{\gamma-\epsilon_{y}}$ divides $Z^{2}$. Since $\epsilon_{y}=\gamma \bmod 2$, we know that $\gamma-\epsilon_{y}$ is even and for $p \geq 1$, nonnegative. Using, for instance, the factorizations of $\beta^{\left(\gamma-\epsilon_{y}\right) / 2}$ and $Z$ into primes, we deduce that $\beta^{\left(\gamma-\epsilon_{y}\right) / 2}$ divides $Z$. Consequently, there exists an integer $X$ such that

$$
Y \cdot \beta^{\epsilon_{y}}=X^{2} \quad \text { and } \quad Z=X \cdot \beta^{\left(\gamma-\epsilon_{y}\right) / 2} .
$$

Now, the assumption $\beta^{p-1} \leq Y<\beta^{p}$ is equivalent to

$$
\beta^{\left(p-1+\epsilon_{y}\right) / 2} \leq X<\beta^{\left(p+\epsilon_{y}\right) / 2},
$$

while the same assumption on $Z$ is equivalent to $\beta^{p-1-\left(\gamma-\epsilon_{y}\right) / 2} \leq X<\beta^{p-\left(\gamma-\epsilon_{y}\right) / 2}$. The latter interval contains the former because $p-1 \leq \delta \leq p$. Hence, $N$ is the number of integers $X$ satisfying (4) and whose square is an integer multiple of $\beta^{\epsilon_{y}}$. We distinguish between the two cases $\epsilon_{y}=0$ and $\epsilon_{y}=1$.

If $\epsilon_{y}=0$, then $N$ is the number of integers $X$ satisfying (4). Consequently, $N=\left\lceil\beta^{p / 2}\right\rceil-\left\lceil\beta^{(p-1) / 2}\right\rceil$ (using either Lemma 1 with $c=1$, or $[4,(3.12)]$ ).

If $\epsilon_{y}=1$, then $X^{2}$ is a multiple of $\beta$ : When $\beta$ has linear factors only (like $\beta=2$ or $\beta=10=2 \cdot 5$ ), this implies that $X$ is a multiple of $\beta$. In this case, $N$ is the number of integers $X$ that are multiples of $\beta$ and satisfy $\beta^{p / 2} \leq X<\beta^{(p+1) / 2}$. Hence, using Lemma $1, N=\left\lceil\beta^{(p-1) / 2}\right\rceil-\left\lceil\beta^{(p-2) / 2}\right\rceil$. Assume now that $\beta=2^{q}$ for some positive integer $q$. If $q$ is even, then $2^{q}$ divides $X^{2}$ implies $2^{q / 2}$ divides $X$ so that we take the number of $X \mathrm{~s}$ being an integer multiple of $2^{q / 2}$. Lemma 1 thus gives $N=\left\lceil 2^{q p / 2}\right\rceil-\left\lceil 2^{q(p-1) / 2}\right\rceil$. If $q$ is odd, then $Y \cdot 2=\left(X \cdot 2^{-\lfloor q / 2\rfloor}\right)^{2}$, which means that $X \cdot 2^{-\lfloor q / 2\rfloor}$ is even. Hence, we keep all the $X$ s that are an integer multiple of $2^{1+\lfloor q / 2\rfloor}$. Using Lemma 1 , this gives $N=\left\lceil 2^{(q p-1) / 2}\right\rceil-$ $\left\lceil 2^{(q(p-1)-1) / 2}\right\rceil$.

For a fixed $e_{y}$, using Theorem 2, one can count the number of input floating-point numbers $y$ whose square root is an exact point. We give below the number $N$ of exact points for the basic formats of the IEEE 754-2008 standard.

\begin{tabular}{|c|c|c|c|c|}
\hline $\begin{array}{c}\text { Format } \\
p\end{array}$ & binary16 & binary32 & binary64 & binary128 \\
& 11 & 24 & 53 & 113 \\
\hline \hline$\epsilon_{y}=0$ & 14 & 1199 & $\approx 2.78 \cdot 10^{7}$ & $\approx 2.98 \cdot 10^{16}$ \\
\hline$\epsilon_{y}=1$ & 9 & 849 & $\approx 1.97 \cdot 10^{7}$ & $\approx 2.11 \cdot 10^{16}$ \\
\hline
\end{tabular}

Also, for a fixed exponent $e_{y}$, one can see from Theorem 2 that the number of exact points for the square root function is $\Theta\left(\beta^{p / 2}\right)$, when the radix $\beta$ is either $2^{q}$ or 10 (it is said that $u(p)=\Theta(v(p))$ if there exist positive constants $c_{1}, c_{2}$, and $p_{0}$ such that $0 \leq c_{1} v(p) \leq u(p) \leq c_{2} v(p)$ for all $p \geq p_{0}$, see, for instance, Graham et al. [4, p. 448] for more details on the $\Theta$ notation). Except for small precisions, Theorem 2 implies, therefore, that it can be regarded as impractical to enumerate the exact points for the square root. It also shows that when computing the square root of a floating-point number, the probability of that square root being an exact point is very small (it vanishes as $p$ increases). This property may be taken into account when tuning a square root algorithm.

\begin{tabular}{|c|c|c|c|}
\hline $\begin{array}{c}\text { Format } \\
p\end{array}$ & $\begin{array}{c}\text { decimal32 } \\
7\end{array}$ & $\begin{array}{c}\text { decimal64 } \\
16\end{array}$ & $\begin{array}{c}\text { decimal128 } \\
34\end{array}$ \\
\hline \hline$\epsilon_{y}=0$ & 2163 & $\approx 6.84 \cdot 10^{7}$ & $\approx 6.84 \cdot 10^{16}$ \\
\hline$\epsilon_{y}=1$ & 683 & $\approx 2.16 \cdot 10^{7}$ & $\approx 2.16 \cdot 10^{16}$ \\
\hline
\end{tabular}

\section{Reciprocal Square Root}

\subsection{Midpoints for Reciprocal Square Root}

Theorem 3. Let $y \in \mathbb{F}_{\beta, p}$ be positive and let $\delta_{y}$ denote $e_{y} \bmod 2$. If $\beta=2^{q}\left(q \in \mathbb{N}_{>0}\right)$, then $1 / \sqrt{y} \notin \mathbf{M I}_{\beta, p}$. If $\beta=10$, one has $1 / \sqrt{y} \in \mathbb{M}_{\beta, p}$ if and only if the integral significand $Y$ of $y$ has the form

$$
Y=2^{3 p-\delta_{y}+1} \cdot 5^{3 p-2 \ell-\delta_{y}-1},
$$

with $\ell \in \mathbb{N}$ such that $\ell \leq\left(3 p-\delta_{y}-1\right) / 2$ and

$$
\begin{cases}2 \cdot 10^{p-1}<5^{\ell}<2 \cdot 10^{p-1 / 2}, & \text { if } e_{y} \text { is odd } \\ 2 \cdot 10^{p-1 / 2}<5^{\ell}<2 \cdot 10^{p}, & \text { if } e_{y} \text { is even. }\end{cases}
$$

Proof. Let $z=1 / \sqrt{y}$ and assume $z \in \mathbb{M}_{\beta, p}$. Let $y=$ $Y \cdot \beta^{e_{y}-p+1}$ and $z=(Z+1 / 2) \cdot \beta^{e_{z}-p+1}$ be the normalized representations of $y$ and $z$. From $y z^{2}=1$, we deduce that

$$
Y(2 Z+1)^{2}=4 \cdot \beta^{-e_{y}-2 e_{z}+3 p-3} .
$$

Since $z$ is a midpoint, one has $\beta^{e_{z}}<z<\beta^{e_{z}+1}$, and so, $\beta^{-2 e_{z}-2}<y<\beta^{-2 e_{z}}$. From this, one may check that

$$
-e_{y}-2 e_{z}=2-\delta_{y}, \quad \delta_{y}=e_{y} \bmod 2 .
$$

Hence, we obtain from (6) and (7):

$$
Y(2 Z+1)^{2}=4 \cdot \beta^{3 p-\delta_{y}-1} .
$$

When $\beta=2^{q}$, (8) has no solution, since the right-hand side of the equality is a power of two, while the left-hand side has an odd factor $(2 Z+1)^{2}$.

Let us now consider the case where $\beta=10$. Equation (8) then becomes

$$
Y(2 Z+1)^{2}=2^{3 p-\delta_{y}+1} \cdot 5^{3 p-\delta_{y}-1} .
$$

Since $2 Z+1$ is odd, we deduce from (9) that $2 Z+1=5^{\ell}$ for some $\ell \in \mathbb{N}$. Hence,

$$
Y=2^{3 p-\delta_{y}+1} \cdot 5^{3 p-2 \ell-\delta_{y}-1},
$$

and it remains to prove the bounds on $\ell$. Since $Y$ is an integer, we have $3 p-2 \ell-\delta_{y}-1 \geq 0$, and the first bound $\ell \leq\left(3 p-\delta_{y}-1\right) / 2$ follows. To prove the bounds in (5), note first that $10^{e_{y}} \leq y<10^{e_{y}+1}$ and (7) gives $10^{e_{z}+\left(1-\delta_{y}\right) / 2}<$ $z=1 / \sqrt{y} \leq 10^{e_{z}+1-\delta_{y} / 2}$. Then, using

$$
z=(Z+1 / 2) \cdot 10^{e_{z}-p+1},
$$


TABLE 2

Integral Significands $Y$ of $y \in \mathbb{F}_{10, p}$ Such That $1 / \sqrt{y} \in \mathbb{M I}_{10, p}$ for the Decimal Formats of the IEEE 754-2008 Standard [5]

\begin{tabular}{|c|c|c|}
\hline Format & Integral significand $Y$ & $e_{y}$ \\
\hline \hline $\begin{array}{c}\text { decimal32 } \\
(p=7)\end{array}$ & $2^{22} \cdot 5^{0}=4194304$ & even \\
\hline decimal64 & $2^{48} \cdot 5^{2}=7036874417766400$ & odd \\
$(p=16)$ & $2^{49} \cdot 5^{1}=2814749767106560$ & even \\
\hline decimal128 & $2^{102} \cdot 5^{4}$ & odd \\
& $=3169126500570573503741758013440000$ & \\
$(p=34)$ & $2^{103} \cdot 5^{3}$ & even \\
\hline
\end{tabular}

we obtain

$$
2 \cdot 10^{p-\left(\delta_{y}+1\right) / 2}<2 Z+1=5^{\ell} \leq 2 \cdot 10^{p-\delta_{y} / 2} .
$$

In fact, the upper bound is strict, for $5^{\ell}$ is an odd integer, while $2 \cdot 10^{p-\delta_{y} / 2}$ is either an even integer $\left(\delta_{y}=0\right)$ or an irrational number $\left(\delta_{y}=1\right)$. Conversely, let

$$
Y=2^{3 p-\delta_{y}+1} \cdot 5^{3 p-2 \ell-\delta_{y}-1},
$$

with $\ell$ as in (5), and let $z=1 / \sqrt{y}$. From (8), we deduce that $y=2^{2 p-2 e_{z}} \cdot 5^{2 p-2 \ell-2 e_{z}-2}$ and

$$
z=\left(\left(5^{\ell}-1\right) / 2+1 / 2\right) \cdot 10^{1-p+e_{z}} .
$$

Now $2 \cdot 10^{p-1}<5^{\ell}<2 \cdot 10^{p}$ implies $10^{p-1} \leq\left(5^{\ell}-1\right) / 2<$ $10^{p}$, and thus, $z \in \mathbb{M}_{10, p}$.

To find in radix 10 the significands $Y$ of all the inputs $y$ such that $1 / \sqrt{y}$ is a midpoint, it suffices to find the at most two $\ell \in \mathbb{N}$ such that $2 \cdot 10^{p-1}<5^{\ell}<2 \cdot 10^{p}$, and to determine from the bounds (5) whether $e_{y}$ is even or odd. Table 2 gives the integral significands $Y$ and the parity of the exponent $e_{y}$ such that $z=1 / \sqrt{y}$ is a midpoint in the basic decimal formats of IEEE 754-2008.

Note that for radices different from 10 or a power of two, we do not have general results (which is in contrast with square root; see Section 2.1). Equation (8) may have solutions; for example, in radix 3 with $p=6$, one may check that $\left(Y, Z, \delta_{y}\right)=(324,364,1)$ satisfies (8) and gives a midpoint for the reciprocal square root.

\subsection{Exact Points for Reciprocal Square Root}

The following theorem gives a characterization of the exact points of the square root reciprocal when the radix is a prime number (which includes the most frequent case $\beta=2$ ) and also when the radix is a positive integer power of two. The case $\beta=10$ is treated separately in Theorem 5 .

Theorem 4. Let $y \in \mathbb{F}_{\beta, p}$ be positive. Then,

- for $\beta$ a prime number, one has $1 / \sqrt{y} \in \mathbb{F}_{\beta, p}$ if and only if $y=\beta^{2 k}$ with $k \in \mathbb{Z}$;

- for $\beta=2^{q}\left(q \in \mathbb{N}_{>0}\right)$, one has $1 / \sqrt{y} \in \mathbb{F}_{\beta, p}$ if and only if $y=2^{2 k}$ with $k \in \mathbb{Z}$.

Proof. Taking $z=1 / \sqrt{y}$, note first that (7) still holds. Now assume that $z \in \mathbb{F}_{\beta, p}$ and let $Y$ and $Z$ be the integral significands of $y$ and $z$. From $y z^{2}=1$ and (7), we deduce

$$
Y Z^{2}=\beta^{3 p-\delta_{y}-1} \text {. }
$$

If $\beta$ is prime, we deduce from (10) that $Z=\beta^{\ell}$ for some $\ell \in \mathbb{N}$. Hence, $Y=\beta^{3 p-\delta_{y}-1-2 \ell}$ and using (7), $y=$ $\beta^{2\left(p-1-e_{z}-\ell\right)}$ is indeed an even power of $\beta$. Conversely, if $y=\beta^{2 k}$, then $z=\beta^{-k}$ is in $\mathbb{F}_{\beta, p}$.

If $\beta=2^{q}$ with $q \in \mathbb{N}_{>0}$, we deduce from (10) that $Z=$ $2^{\ell}$ for some $\ell \in \mathbb{Z}$, and similarly to the previous case, we find $y=2^{2\left(q\left(p-1-e_{z}\right)-\ell\right)}$, which is an even power of two. Conversely, if $y=2^{2 k}$, then $z=2^{-k}$. Since any integral power of two is representable in $\mathbb{F}_{2^{q}, p}$, we conclude that $z$ is an exact point.

All the floating-point numbers $y$ such that $1 / \sqrt{y}$ is an exact point can be deduced from the ones lying in the interval $\left[1, \beta^{2}\right)$. In radix $2^{q}$, Theorem 4 implies that at most $q$ values of $y$ in $\left[1,2^{2 q}\right)$ suffice to characterize the exact points for the reciprocal square root. In radix $16=2^{4}$, for instance, the only exact points for input values $y \in[1,256)$ are:

\begin{tabular}{|c||c|c|c|c|}
\hline$y$ & 1 & 4 & 16 & 64 \\
\hline $1 / \sqrt{y}$ & 1 & $1 / 2=0.8_{16}$ & $1 / 4=0.4_{16}$ & $1 / 8=0.2_{16}$ \\
\hline
\end{tabular}

Theorem 5. Let $y \in \mathbb{F}_{10, p}$ be positive and let $\delta_{y}$ denote $e_{y} \bmod 2$. One has $1 / \sqrt{y} \in \mathbb{F}_{10, p}$ if and only if either $y=10^{-2 e_{z}}$ or the integral significand $Y$ of $y$ differs from $10^{p-1}$ and has the form

$$
Y=2^{3 p-1-\delta_{y}-2 k} \cdot 5^{3 p-1-\delta_{y}-2 \ell},
$$

with $k, \ell \in \mathbb{N}$ such that $0 \leq k, \ell \leq\left(3 p-1-\delta_{y}\right) / 2$.

Proof. Let $z=1 / \sqrt{y}$ and assume $z \in \mathbb{F}_{10, p}$. If $z=10^{e_{z}}$, then obviously, $y=10^{-2 e_{z}}$. On the other hand, $z$ must differ from the irrational number $10^{e_{z}+1 / 2}$. Hence, we now assume that $z \in\left(10^{e_{z}}, 10^{e_{z}+1 / 2}\right) \cup\left(10^{e_{z}+1 / 2}, 10^{e_{z}+1}\right)$. This implies that $y \in\left(10^{-2 e_{z}-2}, 10^{-2 e_{z}-1}\right) \cup\left(10^{-2 e_{z}-1}, 10^{-2 e_{z}}\right)$. Therefore, $y$ is not a power of 10 and its normalized representation $y=Y \cdot 10^{e_{y}-p+1}$ is such that $Y \neq 10^{p-1}$. Note now that (7) and (10) still hold here so that $y z^{2}=1$ implies that $Y Z^{2}=10^{3 p-1-\delta_{y}}$. In particular, $Z$ must have the form $Z=2^{k} \cdot 5^{\ell}$ for some $k, \ell$ in $\mathbb{N}$. Thus,

$$
Y=2^{3 p-1-\delta_{y}-2 k} \cdot 5^{3 p-1-\delta_{y}-2 \ell},
$$

where since $Y$ is an integer, $0 \leq k, \ell \leq\left(3 p-1-\delta_{y}\right) / 2$.

Conversely, the case $y=10^{-2} e_{z}$ being straightforward, let $Y=2^{3 p-1-\delta_{y}-2 k} \cdot 5^{3 p-1-\delta_{y}-2 \ell}$ be the integral significand of $y$ such that $10^{p-1}<Y<10^{p}$, and let $z=1 / \sqrt{y}$. Using (7) further leads to $z=2^{k} \cdot 5^{\ell} \cdot 10^{e_{z}-p+1}$. One has $2^{k} \cdot 5^{\ell} \in \mathbb{N}$ and from $10^{p-1}<Y<10^{p}$, we get $10^{p-\left(1+\delta_{y}\right) / 2}<2^{k} \cdot 5^{\ell}<$ $10^{p-\delta_{y} / 2}$. Hence, $z \in \mathbb{F}_{10, p}$.

Enumerating the integral significands $Y=2^{3 p-1-\delta_{y}-2 k}$. $5^{3 p-1-\delta_{y}-2 \ell}$ with $k, \ell \in \mathbb{N}$ such that $0 \leq k, \ell \leq\left(3 p-1-\delta_{y}\right) / 2$ and $10^{p-1}<Y<10^{p}$ is easily done by a simple program. Table 3 gives all the integral significands $Y$ of $y$, and the parity of the exponent $e_{y}$, such that $1 / \sqrt{y}$ is a floating-point number too, in the decimal32 format (see also Table 8 in the Appendix for the decimal64 format).

For the basic decimal formats of the IEEE 754-2008, the table below gives the number of significands $Y$ such that $1 / \sqrt{y}$ is an exact point, with respect to the parity $\delta_{y}$ of the exponent of $y$.

\begin{tabular}{|c|c|c|c|}
\hline Format & decimal32 & decimal64 & decimal128 \\
$p$ & 7 & 16 & 34 \\
\hline \hline$\delta_{y}=0$ & 9 & 17 & 37 \\
\hline$\delta_{y}=1$ & 7 & 17 & 36 \\
\hline
\end{tabular}


TABLE 3

Integral Significands $Y$ of $y \in \mathbb{F}_{10,7}$ Such That $1 / \sqrt{y} \in \mathbb{F}_{10,7}$

\begin{tabular}{|cl|l|c|}
\hline$Y$ & $1 / \sqrt{Y \cdot 10^{\delta_{y}-p+1}}$ & $e_{y}$ \\
\hline \hline $2^{6} \cdot 5^{6}=1000000$ & $1.000000 \cdot 10^{0}$ & even \\
\hline $2^{20} \cdot 5^{0}=1048576$ & $9.765625 \cdot 10^{-1}$ & even \\
$2^{18} \cdot 5^{2}=6553600$ & $3.906250 \cdot 10^{-1}$ & even \\
$2^{16} \cdot 5^{2}=1638400$ & $7.812500 \cdot 10^{-1}$ & even \\
$2^{12} \cdot 5^{4}=2560000$ & $6.250000 \cdot 10^{-1}$ & even \\
$2^{8} \cdot 5^{6}=4000000$ & $5.000000 \cdot 10^{-1}$ & even \\
$2^{4} \cdot 5^{8}$ & $=6250000$ & $4.000000 \cdot 10^{-1}$ & even \\
$2^{2} \cdot 5^{8}$ & $=1562500$ & $8.000000 \cdot 10^{-1}$ & even \\
$2^{0} \cdot 5^{10}$ & $=9765625$ & $3.200000 \cdot 10^{-1}$ & even \\
$2^{19} \cdot 5^{1}$ & $=2621440$ & $1.953125 \cdot 10^{-1}$ & odd \\
$2^{15} \cdot 5^{3}$ & $=4096000$ & $1.562500 \cdot 10^{-1}$ & odd \\
$2^{13} \cdot 5^{3}$ & $=1024000$ & $3.125000 \cdot 10^{-1}$ & odd \\
$2^{11} \cdot 5^{5}$ & $=6400000$ & $1.250000 \cdot 10^{-1}$ & odd \\
$2^{9} \cdot 5^{5}$ & $=1600000$ & $2.500000 \cdot 10^{-1}$ & odd \\
$2^{5} \cdot 5^{7}$ & $=2500000$ & $2.000000 \cdot 10^{-1}$ & odd \\
$2^{1} \cdot 5^{9}$ & $=3906250$ & $1.600000 \cdot 10^{-1}$ & odd \\
\hline
\end{tabular}

\section{Positive Integer Powers}

We consider here the function $(x, k) \mapsto x^{k}$ with $x \in \mathbb{R}$ and $k \in \mathbb{N}_{>0}$, assuming that each prime factor appears only once in the prime decomposition of $\beta$, which is the case for $\beta=2$ and $\beta=10$. We provide a sufficient condition for the nonexistence of midpoints in such radices. In the particular case $\beta=2$, the results given in this section can be deduced from Lauter and Lefèvre's study of the power function $(x, y) \mapsto x^{y}$ [8], which shows how to check quickly if $x^{y}$ is a midpoint or an exact point in double precision (binary64 format).

Definition 1. A number fits in $n$ digits exactly in radix $\beta$ if it is a precision-n floating-point number that cannot be exactly represented in precision $n-1$. More precisely, it is a number of the form $x=X \cdot \beta^{e_{x}}$, where $e_{x}, X \in \mathbb{Z}, \beta^{n-1}<|X|<\beta^{n}$, and $X$ is not a multiple of $\beta$.

Lemma 3. Let $k \in \mathbb{N}_{>0}$ be given. If each factor of $\beta$ appears only once in its prime number decomposition (which is true for $\beta$ equal to 2 or 10), and if $x$ fits in $n$ digits exactly, then $x^{k}$ fits in $m$ digits exactly, with $m \in \mathbb{N}$ such that $k(n-1)<m \leq k n$.

Proof. Let $x=X \cdot \beta^{e_{x}}$ be a number that fits in $n$ digits exactly. From $\beta^{n-1}<|X|<\beta^{n}$, it follows that $\beta^{k(n-1)}<\left|X^{k}\right|<\beta^{k n}$. Consequently, there exists $m \in \mathbb{N}$ such that $k(n-1)<$ $m \leq k n$ and $\beta^{m-1}<\left|X^{k}\right|<\beta^{m}$. Moreover, the assumption on the prime factor decomposition of $\beta$ and the fact that $\beta$ does not divide $X$ imply that $X^{k}$ is not a multiple of $\beta$.

An immediate consequence of the previous lemma is the following result:

Theorem 6. Assume that the radix $\beta$ is such that each factor appears only once in its prime number decomposition, and let $p$ be the precision. If $x$ fits in $n$ digits exactly, then $x^{k}$ cannot be a midpoint as soon as $k(n-1)>p$, and it cannot be an exact point as soon as $k(n-1)+1>p$.

Theorem 6 is not helpful when $k$ is small. For large values of $k$, however, it allows to quickly determine the possible midpoints and exact points. For instance, in the binary 64 format $(\beta=2$ and $p=53)$, the only floating-point numbers $x$ such that $x^{10}$ can be an exact point are those that fit in $n$ bits exactly, where $n \leq 6$. For a given value of the exponent, there are at most $2^{6}=64$ such points: it, therefore, suffices to check these 64 values to know all the exact points. By accurately computing $x^{10}$ for these 64 points, we easily find that the exact points for function $x^{10}$ in the binary64 format correspond to the input values of the form $x=X \cdot 2^{e_{x}}$, where $X$ is an integer between 0 and 40 .

\section{The Function $(x, y) \mapsto x /\|y\|_{2}$}

Given $d \in \mathbb{N}_{>0}$, the number of exact points of the function that maps $(x, y) \in \mathbb{R} \times\left(\mathbb{R}^{d} \backslash\{0\}\right)$ to $x /\|y\|_{2}=x / \sqrt{\sum_{1 \leq k \leq d} y_{k}^{2}}$ is huge. Indeed, all the exact points for the division operation, whose number is huge as we will see later in Section 6.2, are exact points for the function $x /\|y\|_{2}$ as well. Therefore, we shall focus here exclusively on midpoints: our aim is to decide whether there exist floating-point inputs $x, y_{1}, \ldots, y_{d} \in \mathbb{F}_{\beta, p}$ such that $x /\|y\|_{2} \in \mathbb{M}_{\beta, p}$. We start with the following theorem, which says that midpoints cannot exist in radix 2.

Theorem 7. Let $x \in \mathbb{F}_{\beta, p}$ and for $d \in \mathbb{N}_{>0}$, let $y$ be a nonzero, $d$-dimensional vector of elements of $\mathbb{F}_{\beta, p}$. If $\beta=2$, then $x /\|y\|_{2} \notin \mathbb{M}_{\beta, p}$.

Proof. Because of the symmetries of the function that maps $(x, y)$ to $x /\|y\|_{2}$, we can restrict to the case where $x$ and all the entries of $y=\left[y_{k}\right]$ are positive. Hence, $x=$ $X \cdot \beta^{e_{x}-p+1}$ and $y_{k}=Y_{k} \cdot \beta^{e_{y_{k}}-p+1}$ for some integers $X$ and $Y_{k}$ such that $\beta^{p-1} \leq X, Y_{k}<\beta^{p}$. Let $z=x /\|y\|_{2}$ and assume that $z$ is a midpoint, that is, $z=(Z+1 / 2)$. $\beta^{e_{z}-p+1}$ for some integer $Z$ in the same range as $X$ and the $Y_{k}$ above. The identity $x^{2}=\|y\|_{2}^{2} z^{2}$ thus becomes:

$$
4 X^{2} \cdot \beta^{2\left(e_{x}-e_{z}+p-1\right)}=\left(\sum_{k} Y_{k}^{2} \cdot \beta^{2 e_{y_{k}}}\right)(2 Z+1)^{2} .
$$

In order to have integers on both sides, it suffices to multiply (11) by $\beta^{-2 e^{*}}$, where $e^{*}=\min _{k} e_{y_{k}}$. This gives

$4 X^{2} \cdot \beta^{2\left(e_{x}-e_{z}-e^{*}+p-1\right)}=\left(\sum_{k} Y_{k}^{2} \cdot \beta^{2\left(e_{y_{k}}-e^{*}\right)}\right)(2 Z+1)^{2}$.

Now, the power of $\beta$ involved in the left-hand side of (12) is itself an integer. This is due to the fact that the integer $e_{x}-e_{z}-e^{*}$ is nonnegative, which can be seen as follows: Since $d \geq 1$ and $y_{k} \geq \beta^{e^{*}}$ for $k=1, \ldots, d$, one has $z \leq x / \beta^{e^{*}}$. Using $x<\beta^{e_{x}+1}$ and $\beta^{e_{z}} \leq z$ (in fact, this lower bound is strict, for $z$ is a midpoint), we deduce that $\beta^{e_{z}}<\beta^{e_{x}-e^{*}+1}$. The exponents on both sides of the latter inequality being integers, we conclude that $e_{z} \leq e_{x}-e^{*}$. When $\beta=2$, (12) becomes

$$
X^{2} \cdot 2^{2\left(e_{x}-e_{z}-e^{*}+p\right)}=\left(\sum_{k} Y_{k}^{2} \cdot 2^{2\left(e_{y_{k}}-e^{*}\right)}\right)(2 Z+1)^{2} .
$$

The left-hand side of (13) is a multiple of the odd integer $(2 Z+1)^{2}$. Since $e_{x}-e_{z}-e^{*}$ is nonnegative, this implies that $X$ is a multiple of $2 Z+1$, and thus, $X \geq 2 Z+1$. However, recalling that $2^{p-1} \leq X, Z<2^{p}$, we have

$$
X<2 Z+1 \text {. }
$$

Hence, a contradiction, which concludes the proof. 
Theorem 7 implies the nonexistence of midpoints in radix $\beta=2$ for a number of important special cases: division $x / y$ (see Corollary 1 ), and thus, reciprocal $1 / y$ as well; reciprocal 2D euclidean norm $1 / \sqrt{x^{2}+y^{2}}$ and 2D-vector normalization $x / \sqrt{x^{2}+y^{2}}$.

However, when $\beta>2$, the function $x /\|y\|_{2}$ does have midpoints and some examples will be given in Section 6.1 for $\beta \in\{3,4,10\}$. Thus, rather than trying to characterize all the midpoints of that general function, we focus from Section 6 to Section 9 on the four special cases just mentioned.

\section{Division}

\subsection{Midpoints for Division}

Concerning midpoints for division, Theorem 7 gives an answer for the far most frequent case in practice: the radix is two, the input precision equals the output precision, and the results are above the underflow threshold. Indeed, choosing $d=1$ in Theorem 7 , we obtain the following corollary:

Corollary 1. In binary arithmetic, the quotient of two floatingpoint numbers cannot be a midpoint in the same precision.

In radix-2 floating-point arithmetic, Corollary 1 can be seen as a consequence of a result presented by Markstein in $[9$, Theorem 8.4, p. 114]. Note that this result only holds when $\beta=2$ and when the input precision is less than or equal to the output precision. Nevertheless, it is sometimes believed that it holds in prime radices: the first example given below shows that this is not the case. The following examples also illustrate the existence of midpoints when $\beta>2$.

- In radix 3, with precision $p=4$,

$$
\frac{28_{10}}{56_{10}}=\frac{1001_{3}}{2002_{3}}=0.1111_{3}+\frac{1}{2} \cdot 3^{-4} .
$$

- In radix 4 , with $p=4$,

$$
\frac{129_{10}}{128_{10}}=\frac{2001_{4}}{2000_{4}}=1.000_{4}+\frac{1}{2} \cdot 4^{-3} .
$$

- In radix 10, midpoint quotients are quite frequent. For instance, when $p=2$, there are 181 midpoints for $X / Y$ with $10 \leq X, Y \leq 99$ (that is, $10 / 16=0.625$ ) and when $p=3$, there are 2,633 cases with $100 \leq X, Y \leq 999$.

We now briefly discuss the case of different input $\left(p_{i}\right)$ and output $\left(p_{o}\right)$ precisions. If $p_{i}>p_{o}$, many quotients can be midpoints, even in radix-2 arithmetic: If $x$ is in precision $p_{i}>p_{o}, x$ can be a midpoint in precision $p_{o}$, which is then the case for the quotient $x / 1$. It is also possible to find less trivial cases. For example, if $x$ and $y$ are binary 64 numbers $\left(p_{i}=53\right)$ with

$$
\begin{aligned}
x= & 1.000000000000000000000000 \\
& 1111111111111111111110100000, \\
y= & 1.1111111111111111111111
\end{aligned}
$$

000000000000000000000000000000 then one has

$$
x / y=0 . \underbrace{100000000000000000000001}_{p_{o}=24} 1,
$$

which is a midpoint in the binary32 floating-point format $\left(p_{o}=24\right)$.

\subsection{Exact Points for Division}

Let $x$ and $y$ be two numbers in $\mathbb{F}_{\beta, p}$, and assume that the quotient $z=x / y$ is also in $\mathbb{F}_{\beta, p}$. Using the normalized representations $x=X \cdot \beta^{e_{x}-p+1}$ and $y=Y \cdot \beta^{e_{y}-p+1}$, then $z$ can be written $z=Z \cdot \beta^{e_{x}-e_{y}+\delta-p}$, with $\delta \in\{0,1\}$. Hence, from $x=y z$, it follows that

$$
\beta^{p-\delta} X=Y Z,
$$

with $\delta \in\{0,1\}$. In other words, if $z$ is an exact point, then (15) must be satisfied. For any radix $\beta,(15)$ has many solutions: for each value of $X$, there is at least the straightforward solution $(X, Y)=\left(Z, \beta^{p-1}\right)$, which corresponds to $x / \beta^{e_{y}}$. As a consequence, the number of exact points of the function $(x, y) \mapsto x / y$ grows at least like $\beta^{p-1}(\beta-1)$ for any given exponents $e_{x}, e_{y}$. This is too large to enumerate all the exact points of division in practice.

\section{ReCIPROCAL}

As we have seen above, except in radix 2, division admits many midpoints. Moreover, whatever the radix is, division also admits a lot of exact points. Consequently, we now focus on a special case, the reciprocal function $y \mapsto 1 / y$, for which more useful results can be obtained.

\subsection{Midpoints for Reciprocal}

Theorem 8. Let $y \in \mathbb{F}_{\beta, p}$ be nonzero. If $\beta=2^{q}\left(q \in \mathbb{N}_{>0}\right)$, then $1 / y \notin \mathbb{M I}_{\beta, p}$. If $\beta=10$, one has $1 / y \in \mathbb{M I}_{\beta, p}$ if and only if the integral significand $Y$ of $y$ has the form

$$
Y=2^{2 p} \cdot 5^{2 p-1-\ell},
$$

with $\ell \in \mathbb{N}$ such that $2 \cdot 10^{p-1}<5^{\ell}<2 \cdot 10^{p}$.

Proof. Without loss of generality, we assume that $y>0$. Let $z=1 / y$. First, one may check that

$$
e_{z}=-e_{y}-1 \text {. }
$$

Now, if $z \in \mathbb{M}_{\beta, p}$, then $z=(Z+1 / 2) \cdot \beta^{e_{z}-p+1}$ for some integer $Z$ such that $\beta^{p-1} \leq Z<\beta^{p}$. Using $y z=1$ thus gives

$$
Y(2 Z+1)=2 \cdot \beta^{2 p-1} .
$$

When $\beta=2^{q}$, (18) has no solution, since the righthand side of the equality is a power of two, while the left-hand side has an odd factor $2 Z+1$. When $\beta=10$, (18) becomes

$$
Y(2 Z+1)=2^{2 p} \cdot 5^{2 p-1} .
$$

As $2 Z+1$ is odd, we deduce from (19) that $2 Z+1$ is a power of five. Also, since $2 \cdot 10^{p-1}<2 Z+1<2 \cdot 10^{p}$, there are at most two such powers of 5. Hence, $y$ is necessarily as in (16). Conversely, if $y=Y \cdot 10^{e_{y}-p+1}$ with $Y$ as in (16), then using (17), $y=5^{-\ell-1} \cdot 10^{-e_{z}+p}$. It follows 
TABLE 4

Integral Significands $Y$ of $y \in \mathbb{F}_{10, p}$ Such That $1 / y \in \mathbb{M}_{10, p}$, for the Decimal Formats of the IEEE 754-2008 Standard [5]

\begin{tabular}{|c|c|}
\hline Format & \multicolumn{1}{|c|}{ Integral significand $Y$} \\
\hline \hline $\begin{array}{c}\text { decimal32 } \\
(p=7)\end{array}$ & $2^{14} \cdot 5^{3}=2048000$ \\
\hline decimal64 & $2^{32} \cdot 5^{8}=1677721600000000$ \\
$(p=16)$ & $2^{32} \cdot 5^{9}=8388608000000000$ \\
\hline decimal128 & $2^{68} \cdot 5^{18}=1125899906842624000000000000000000$ \\
$(p=34)$ & $2^{68} \cdot 5^{19}=5629499534213120000000000000000000$ \\
\hline
\end{tabular}

that $z$ can be written $z=\left(\left(5^{\ell}-1\right) / 2+1 / 2\right) \cdot 10^{e_{z}-p+1}$. Since $\left(5^{\ell}-1\right) / 2$ is an integer, and by hypothesis $10^{p-1} \leq\left(5^{\ell}-1\right) / 2<10^{p}$, we deduce that $z \in \mathbf{M I}_{10, p}$, which concludes the proof.

In radix 10 , there are at most two values of $\ell \in \mathbb{N}$ such that $2 \cdot 10^{p-1}<5^{\ell}<2 \cdot 10^{p}$. Therefore, to determine all inputs $y$ that give a midpoint $1 / y$ for a fixed exponent $e_{y}$, it suffices to find the at most two $\ell$ such that $2 \cdot 10^{p-1}<$ $5^{\ell}<2 \cdot 10^{p}$. This is easily done, even when the precision $p$ is large. Table 4 gives the integral significands $Y$ of the floating-point numbers $y$ such that $1 / y$ is a midpoint, for the decimal formats of the IEEE 754-2008 standard [5].

\subsection{Exact Points for Reciprocal}

For radices either 10 or a positive power of two, the exact points of the reciprocal function can all be enumerated according to the following theorem:

Theorem 9. Let $y \in \mathbb{F}_{\beta, p}$ be nonzero. One has $1 / y \in \mathbb{F}_{\beta, p}$ if and only if the integral significand $Y$ of $y$ satisfies $\beta^{p-1} \leq$ $Y<\beta^{p}$ and

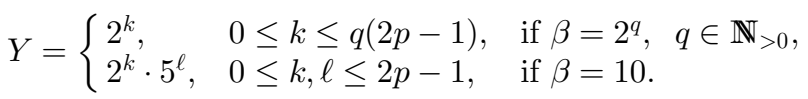

Proof. For the "only if" statement, let $y>0$ in $\mathbb{F}_{\beta, p}$ be given, let $z=1 / y$, and assume that $z \in \mathbb{F}_{\beta, p}$. First, one may check that the exponent of $z$ satisfies $e_{z}=-e_{y}-\delta$ with $\delta \in\{0,1\}$. Then, using the identity $y z=1$ together with the normalized representations $y=Y \cdot \beta^{e_{y}-p+1}$ and $z=Z \cdot \beta^{e_{z}-p+1}$, we get

$$
Y Z=\beta^{2 p-2+\delta}, \quad \beta^{p-1} \leq Y, Z<\beta^{p} .
$$

If $\beta=2^{q}$ for some integer $q \geq 1$, then (20) implies that $Y=2^{k}$ for some integer $k$ such that $0 \leq k \leq q(2 p-1)$. If $\beta=10$, then (20) implies that $Y=2^{k} \cdot 5^{\ell}$ for some integers $k$ and $\ell$ such that $0 \leq k, \ell \leq 2 p-1$.

Let us now prove the "if" statement. If $Y=\beta^{p-1}$, then $y$ is a power of the radix, and thus, $1 / y$ belongs to $\mathbb{F}_{\beta, p}$. If $\beta^{p-1}<Y<\beta^{p}$, then defining $Z=Y^{-1} \cdot \beta^{2 p-1}$, we obtain

$$
1 / y=Z \cdot \beta^{-e_{y}-p}, \quad \beta^{p-1}<Z<\beta^{p} .
$$

To conclude that $1 / y$ belongs to $\mathbb{F}_{\beta, p}$ it remains to show that $Z$ is an integer: If $\beta=2^{q}$ and $Y=2^{k}$, one has $Z=2^{q(2 p-1)-k}$, which is an integer for $k \leq q(2 p-1)$; If $\beta=10$ and $Y=2^{k} \cdot 5^{\ell}$, then $Z=2^{2 p-1-k} \cdot 5^{2 p-1-\ell}$, which is an integer for $k, \ell \leq 2 p-1$. Hence, $Z$ is an integer in both cases, showing that $1 / y$ is indeed an exact point. This concludes the proof.
TABLE 5

Integral Significands $Y$ of $y \in \mathbb{F}_{10,7}$ Such That $1 / y \in \mathbb{F}_{10,7}$

\begin{tabular}{|cc|c|}
\hline$Y$ & $1 / Y$ \\
\hline \hline $2^{0} \cdot 5^{9}$ & $=1953125$ & $5.120000 \cdot 10^{-7}$ \\
$2^{0} \cdot 5^{10}$ & $=9765625$ & $1.024000 \cdot 10^{-7}$ \\
$2^{1} \cdot 5^{9}$ & $=3906250$ & $2.560000 \cdot 10^{-7}$ \\
$2^{2} \cdot 5^{8}$ & $=1562500$ & $6.400000 \cdot 10^{-7}$ \\
$2^{2} \cdot 5^{9}$ & $=7812500$ & $1.280000 \cdot 10^{-7}$ \\
$2^{3} \cdot 5^{8}$ & $=3125000$ & $3.200000 \cdot 10^{-7}$ \\
$2^{4} \cdot 5^{7}$ & $=1250000$ & $8.000000 \cdot 10^{-7}$ \\
$2^{4} \cdot 5^{8}$ & $=6250000$ & $1.600000 \cdot 10^{-7}$ \\
$2^{5} \cdot 5^{7}$ & $=2500000$ & $4.000000 \cdot 10^{-7}$ \\
$2^{6} \cdot 5^{6}$ & $=1000000$ & $1.000000 \cdot 10^{-6}$ \\
$2^{6} \cdot 5^{7}$ & $=5000000$ & $2.000000 \cdot 10^{-7}$ \\
$2^{7} \cdot 5^{6}$ & $=2000000$ & $5.000000 \cdot 10^{-7}$ \\
$2^{8} \cdot 5^{6}$ & $=4000000$ & $2.500000 \cdot 10^{-7}$ \\
$2^{9} \cdot 5^{5}$ & $=1600000$ & $6.250000 \cdot 10^{-7}$ \\
$2^{9} \cdot 5^{6}$ & $=8000000$ & $1.250000 \cdot 10^{-7}$ \\
$2^{10} \cdot 5^{5}$ & $=3200000$ & $3.125000 \cdot 10^{-7}$ \\
$2^{11} \cdot 5^{4}$ & $=1280000$ & $7.812500 \cdot 10^{-7}$ \\
$2^{11} \cdot 5^{5}$ & $=6400000$ & $1.562500 \cdot 10^{-7}$ \\
$2^{12} \cdot 5^{4}$ & $=2560000$ & $3.906250 \cdot 10^{-7}$ \\
$2^{13} \cdot 5^{3}$ & $=1024000$ & $9.765625 \cdot 10^{-7}$ \\
$2^{13} \cdot 5^{4}$ & $=5120000$ & $1.953125 \cdot 10^{-7}$ \\
\hline
\end{tabular}

In radix $16=2^{4}$, for instance, the exact points $1 / y$ with $y$ in the interval $[1,16)$ are listed below.

\begin{tabular}{|c||c|c|c|c|}
\hline$y$ & 1 & 2 & 4 & 8 \\
\hline $1 / y$ & 1 & $1 / 2=0.8_{16}$ & $1 / 4=0.4_{16}$ & $1 / 8=0.2_{16}$ \\
\hline
\end{tabular}

In radix 10 , all the integers $Y=2^{k} \cdot 5^{\ell}$ with $0 \leq k, \ell \leq$ $2 p-1$ and $10^{p-1} \leq Y<10^{p}$ can be enumerated by a simple program, and each one of them gives an exact point. Table 5 gives the 21 integral significands $Y$ such that $1 / y$ is an exact point, in the case of the decimal32 format (see also Table 7 in the Appendix for the decimal64 format).

Furthermore, given an input exponent, the result below provides an explicit formula for the number $N$ of floatingpoint inputs having this exponent and whose reciprocal is a floating-point number.

Theorem 10. For a given exponent $e_{y}$, the number $N$ of positive values $y \in \mathbb{F}_{\beta, p}$ such that $1 / y \in \mathbb{F}_{\beta, p}$ is

$$
N= \begin{cases}q, & \text { if } \beta=2^{q}, \quad q \in \mathbb{N}_{>0}, \\ 2\left\lfloor p \log _{5}(10)\right\rfloor+1, & \text { if } \beta=10 .\end{cases}
$$

Proof. When $\beta=2^{q}$, Theorem 9 says that each exact point corresponds to an integer $k$ such that $2^{q(p-1)} \leq 2^{k}<2^{q p}$ and $0 \leq k \leq q(2 p-1)$. The former condition is equivalent to $q(p-1) \leq k<q p$, and thus, implies the latter. From this, we deduce that the number of possible values of $k$ is $q$ when $\beta=2^{q}$.

When $\beta=10$, Theorem 9 says that each exact point corresponds to a pair of integers $(k, \ell)$ such that

$$
10^{p-1} \leq 2^{k} \cdot 5^{\ell}<10^{p} \quad \text { and } \quad 0 \leq k, \ell \leq 2 p-1 .
$$

The value of $N$ is the number of points $(k, \ell) \in \mathbb{Z}^{2}$ that satisfy those two sets of constraints. Let $\sigma=\log _{5}(2)=$ $0.4306765581 \ldots$ The first set of constraints is equivalent to

$$
(p-1)(1+\sigma) \leq \sigma k+\ell<p(1+\sigma)
$$


It implies in particular that $(p-1)(1+\sigma) \leq \ell<p(1+\sigma)$, which is stronger than $0 \leq \ell \leq 2 p-1$ for $p \geq 2$, since $1+\sigma \approx 1.43$. Hence, $N=\sum_{0 \leq k<2 p} N_{k}$, where $N_{k}$ is the number of integers $\ell$ satisfying (22) for a given $k$.

Recalling that half-open real intervals $[a, b)$ such that $a \leq b$ contain exactly $\lceil b\rceil-\lceil a\rceil$ integers $[4$, p. 74], we deduce that for $0 \leq k<2 p$,

$$
\begin{aligned}
N_{k} & =\lceil p(1+\sigma)-\sigma k\rceil-\lceil(p-1)(1+\sigma)-\sigma k\rceil \\
& =\lceil(p-k) \sigma\rceil-\lceil(p-k-1) \sigma\rceil+1 .
\end{aligned}
$$

Consequently, the sum $\sum_{0 \leq k<2 p} N_{k}$ telescopes to $2 p+\lfloor p \sigma\rfloor+\lceil p \sigma\rceil$. Since the integer $p$ is nonzero and $\sigma$ is irrational, $p \sigma$ cannot be an integer. Hence, $\lceil p \sigma\rceil=\lfloor p \sigma\rfloor+1$, which leads to $N=2\lfloor p(1+\sigma)\rfloor+1$.

According to Theorem 10, when $\beta=2^{q}$, the number $N$ of different integral significands leading to an exact point is $q$. In radix 10, we have $N=\Theta(p)$, which confirms the fact that the midpoints for the reciprocal can be easily enumerated, even when the precision $p$ is large. This is in contrast with the exact points of square root in radix 10 or $2^{q}$, whose number is exponential in $p$ (see Section 2.2). For the decimal formats of IEEE 754-2008, the corresponding values of $N$ are listed below.

\begin{tabular}{|c|c|c|c|}
\hline $\begin{array}{c}\text { Format } \\
p\end{array}$ & decimal32 & decimal64 & decimal128 \\
\hline & 21 & 45 & 34 \\
\hline \hline$N$ & 21 & 97 \\
\hline
\end{tabular}

\section{Reciprocal 2D Euclidean Norm}

Given a $d$-dimensional vector $y$ with entries in $\mathbb{F}_{2, p}$, we know from Theorem 7 that $z=1 /\|y\|_{2}$ cannot be a midpoint in radix 2 . In this section, we focus on the $2 \mathrm{D}$ case, studying the midpoints and the exact points of the reciprocal 2D euclidean norm, in radices $2^{q}$ and 10 . In radix 10, our study relies on the representation of products of the form $2^{r} \cdot 5^{s}$ as sums of two squares $a^{2}+b^{2}$, where $a, b \in \mathbb{N}$. Thus, we first explain in Section 8.1 the method we used for enumerating all the representations of such a product as the sum of two integer squares. Then, midpoints and exact points are studied in Sections 8.2 and 8.3, respectively.

\subsection{Decomposing $2^{r} \cdot 5^{s}$ into Sums of Two Squares}

Decomposing an integer into sums of two squares is a wellstudied problem in the mathematical literature (see, for instance, Wagon [13] and the references therein). In our particular case of interest, we deduce the following theorem that allows us to compute all the decompositions of $2^{r} \cdot 5^{s}$ as sums of two squares. The proof of Theorem 11 relies on the uniqueness of the decomposition of a number into prime factors in the ring of Gaussian integers ${ }^{1} \mathbb{Z}[i]$ (see, for instance, Everest and Ward [3, chap. 2] for more details on this topic).

Theorem 11. Let $r, s \in \mathbb{N}$ be given, and assume $k \in \mathbb{N}$. All the unordered pairs $\{a, b\}$ with $a, b \in \mathbb{N}$ and $a^{2}+b^{2}=2^{r} \cdot 5^{s}$ are given by $a=|\Re(c)|$ and $b=|\Im(c)|$ with

$$
c=2^{\lfloor r / 2\rfloor}(1+i)^{r \bmod 2}(2+i)^{k}(2-i)^{s-k}, 0 \leq k<\lceil(s+1) / 2\rceil .
$$

1. $\mathbb{Z}[i]$ is the set of the numbers of the form $a+i b$, where $a$ and $b$ are integers.
In particular, there are exactly $\lceil(s+1) / 2\rceil$ different decompositions of $2^{r} \cdot 5^{s}$ as the sum of two squares.

Proof. Let us assume that $2^{r} \cdot 5^{s}=a^{2}+b^{2}$. Since the decomposition of $2^{r} \cdot 5^{s}$ into prime factors in $\mathbb{Z}[i]$ is unique apart from multiplications by \pm 1 or $\pm i$, one has $2^{r} \cdot 5^{s}=$ $\delta_{0}(1+i)^{r}(1-i)^{r}(2+i)^{s}(2-i)^{s}$ with $\delta_{0} \in\{ \pm 1, \pm i\}$. On the other hand, one has $a^{2}+b^{2}=(a+i b)(a-i b)$; hence, by uniqueness of the decomposition into prime factors, it follows that $a+i b=\delta_{1}(1+i)^{k_{1}}(1-i)^{k_{2}}(2+i)^{k_{3}}(2-i)^{k_{4}}$ for some $k_{1}, k_{2}, k_{3}, k_{4} \in \mathbb{N}$ and $\delta_{1} \in\{ \pm 1, \pm i\}$. Then, one has

$$
\begin{aligned}
a^{2} & +b^{2} \\
& =\delta_{1} \overline{\delta_{1}}(1+i)^{k_{1}+k_{2}}(1-i)^{k_{1}+k_{2}}(2+i)^{k_{3}+k_{4}}(2-i)^{k_{3}+k_{4}},
\end{aligned}
$$

and from $2^{r} \cdot 5^{s}=a^{2}+b^{2}$, we deduce that $k_{1}+k_{2}=r$ and $k_{3}+k_{4}=s$. Moreover, distinguishing two cases corresponding to the parity of $r$, it can be checked that

$$
(1+i)^{k_{1}}(1-i)^{k_{2}}=\delta_{2} \cdot 2^{\lfloor r / 2\rfloor}(1+i)^{r \bmod 2},
$$

with $\delta_{2} \in\{ \pm 1, \pm i\}$. Hence, we obtain

$$
a+i b=\delta \cdot 2^{\lfloor r / 2\rfloor}(1+i)^{r \bmod 2}(2+i)^{k}(2-i)^{s-k},
$$

for some $\delta \in\{ \pm 1, \pm i\}$ and $k \in \mathbb{N}$ such that $0 \leq k \leq s$. Since $a, b \geq 0$, we deduce that necessarily $a=|\Re(c)|$ and $b=|\Im(c)|$ with $c=2^{\lfloor r / 2\rfloor}(1+i)^{r \bmod 2}(2+i)^{k}(2-i)^{s-k}$. However, since both $c$ and $\bar{c}=2^{\lfloor r / 2\rfloor}(1-i)^{r \bmod 2}(2-$ $i)^{k}(2+i)^{s-k}$ lead to the same unordered pair $\{a, b\}$, there are at most $\lceil(s+1) / 2\rceil$ such unordered pairs $\{a, b\}$. This implies that we only need the assumption $0 \leq k<$ $\lceil(s+1) / 2\rceil$ for $k$.

Conversely, if $a=|\Re(c)|$ and $b=|\Im(c)|$ with $c=$ $2^{\lfloor r / 2\rfloor}(1+i)^{r \bmod 2}(2+i)^{k}(2-i)^{s-k}$, then

$$
a+i b=\delta 2^{\lfloor r / 2\rfloor}(1+i)^{r \bmod 2}(2+i)^{k}(2-i)^{s-k}
$$

with $\delta \in\{ \pm 1, \pm i\}$. Then, one can check that $a^{2}+b^{2}=$ $(a+i b)(a-i b)=2^{r} \cdot 5^{s}$.

By uniqueness of the factorization into primes in $\mathbb{Z}[i]$, it can be shown that if we take $k_{1} \neq k_{2}$ with $0 \leq k_{1}$, $k_{2}<\lceil(s+1) / 2\rceil$, then the corresponding unordered pairs $\left\{a_{1}, b_{1}\right\}$ and $\left\{a_{2}, b_{2}\right\}$ are necessarily different. Hence, there are exactly $\lceil(s+1) / 2\rceil$ unordered pairs $\{a, b\}$.

For later use, we also state the following corollary of Theorem 11:

Corollary 2. Given $r \in \mathbb{N}$, the unique decomposition of $2^{r}$ as a sum of two integer squares is

$$
2^{r}= \begin{cases}0^{2}+\left(2^{r / 2}\right)^{2}, & \text { if } r \text { is even, } \\ \left(2^{(r-1) / 2}\right)^{2}+\left(2^{(r-1) / 2}\right)^{2}, & \text { if } \mathrm{r} \text { is odd }\end{cases}
$$

\subsection{Midpoints for Reciprocal 2D Norm}

Theorem 12 below can be used to determine all the midpoints of the reciprocal 2D-norm function with exponent $e_{z}$.

Theorem 12. Let $x, y \in \mathbb{F}_{\beta, p}$ be such that $(x, y) \neq(0,0)$, and let $z=1 / \sqrt{x^{2}+y^{2}}$. If $\beta=2^{q}\left(q \in \mathbb{N}_{>0}\right)$, then $z \notin \mathbb{M I}_{\beta, p}$. If $\beta=10$, one has $z \in \mathbb{M}_{\beta, p}$ if and only if $z$ has the form 


$$
\left(\frac{5^{\ell}-1}{2}+\frac{1}{2}\right) \cdot 10^{e_{z}-p+1}
$$

with $e_{z} \in \mathbb{Z}$ and $\ell \in \mathbb{N}$ such that $2 \cdot 10^{p-1}<5^{\ell}<2 \cdot 10^{p}$.

Proof. Let $z=1 / \sqrt{x^{2}+y^{2}}$ be a midpoint, with $x, y \in \mathbb{F}_{\beta, p}$. Without loss of generality, we assume that $z$ is in $[1, \beta)$, and since $z$ is a midpoint, then one has $1<z<\beta$. Let us also assume that $x \geq y \geq 0$, which implies

$$
\frac{1}{\sqrt{2} x} \leq \frac{1}{\sqrt{x^{2}+y^{2}}} \leq \frac{1}{x}
$$

Denoting by $e_{x}$ and $e_{y}$ the exponents of $x$ and $y$, respectively, from (23), it follows that $\beta^{-e_{x}-2}<z \leq \beta^{-e_{x}}$, and since $1<z<\beta$, necessarily $e_{x} \in\{-1,-2\}$. Writing $z=(Z+1 / 2) \cdot \beta^{-p+1}$, with $Z \in \mathbb{N}$ such that $\beta^{p-1} \leq$ $Z<\beta^{p}$, from $\left(x^{2}+y^{2}\right) z^{2}=1$, we deduce

$$
\left(X^{2} \cdot \beta^{2 e_{x}-2 e_{y}}+Y^{2}\right)(2 Z+1)^{2}=4 \cdot \beta^{4 p-2 e_{y}-4} .
$$

Note that $x \geq y$ implies that $e_{x} \geq e_{y}$, so that the left-hand side of (24) is indeed in $\mathbb{N}$. When $\beta=2^{q}$, (24) has no solution, since the right-hand side of the equality is a power of two, while the left-hand side has an odd factor. When $\beta=10$, (24) becomes

$$
\left(X^{2} \cdot 10^{2 e_{x}-2 e_{y}}+Y^{2}\right)(2 Z+1)^{2}=2^{4 p-2 e_{y}-2} \cdot 5^{4 p-2 e_{y}-4} .
$$

Then, one has necessarily $2 Z+1=5^{\ell}$ with $\ell \in \mathbb{N}$. The bounds on $5^{\ell}$ follow from $10^{p-1} \leq Z \leq 10^{p}-1$. Conversely, if $z$ has the form given in Theorem 12, it is clearly a midpoint.

For instance, in the decimal32 format of IEEE 754-2008 $(p=7)$, function $1 / \sqrt{x^{2}+y^{2}}$ has only one midpoint in $[1,10)$, namely, $z=4.8828125$. This midpoint corresponds to $5^{10}=9765625$, which is the only power of five in the interval $\left(2 \cdot 10^{6}, 2 \cdot 10^{7}\right)$. All the other midpoints of the function are obtained by multiplying 4.8828125 by an integral power of 10 .

Theorem 12 can only be used to determine the midpoints of the reciprocal norm function. Given such a midpoint $z$, let us now show how to find $x$ and $y$ in $\mathbb{F}_{10, p}$ such that $z=1 / \sqrt{x^{2}+y^{2}}$. For this, we shall use the following trivial lemma:

Lemma 4. Let a be in $\mathbb{Q}$. One has $a^{2} \in \mathbb{N}$ if and only if $a \in \mathbb{Z}$.

As in the proof of Theorem 12, let us assume that $1<$ $z<10$ and $x \geq y \geq 0$, which implies that $e_{x} \in\{-1,-2\}$. We denote by $X$ and $Y$ the integral significands of $x$ and $y$, respectively. From (25), we can deduce that $X$ and $Y$ must satisfy

$$
2^{4 p+2} \cdot 5^{4 p-2 \ell}=\left(X \cdot 10^{e_{x}+2}\right)^{2}+\left(Y \cdot 10^{e_{y}+2}\right)^{2} .
$$

From $2 \cdot 10^{p-1}<5^{\ell}<2 \cdot 10^{p}$, one has $5^{4 p-2 \ell} \in \mathbb{N}$. Since, moreover, $e_{x} \in\{-1,-2\}$, necessarily $X \cdot 10^{e_{x}+2} \in \mathbb{N}$, and $Y^{2} \cdot 10^{2\left(e_{y}+2\right)}$ is also in $\mathbb{N}$. Since $Y \cdot 10^{e_{y}+2}$ is a nonnegative rational number whose square is a natural integer, it follows from Lemma 4 that $Y \cdot 10^{e_{y}+2} \in \mathbb{N}$. Hence, we know that $X \cdot 10^{e_{x}+2}$ and $Y \cdot 10^{e_{y}+2}$ both necessarily belong to $\mathbb{N}$.

As a consequence, to find all inputs $(X, Y)$ that give a midpoint for the function $1 / \sqrt{x^{2}+y^{2}}$, we know from (26) that we need to find all the decompositions of the at most
TABLE 6

Floating-Point Numbers $x, y \in \mathbb{F}_{10,7}$ with $X \geq Y$ Such That $z=1 / \sqrt{x^{2}+y^{2}}$ Is a Midpoint, with $10^{-8} \leq z<10^{-7}$

\begin{tabular}{|c|c|c|}
\hline$x$ & $y$ & $z=1 / \sqrt{x^{2}+y^{2}}$ \\
\hline \hline 1966080 & 573440.0 & \\
1638400 & 1228800 & $4.8828125 \times 10^{-7}$ \\
1916928 & 720896.0 & \\
2048000 & 0 & \\
\hline
\end{tabular}

two integers $2^{4 p+2} \cdot 5^{4 p-2 \ell}$ as the sum of two squares. We used Theorem 11 to build all values $x$ and $y, x \geq y$, such that $1 / \sqrt{x^{2}+y^{2}}$ is a midpoint, for the decimal formats of the IEEE 754-2008 standard. For the decimal32 format, all the pairs of floating-point numbers $(x, y)$ for which $1 / \sqrt{x^{2}+y^{2}}$ is a midpoint can be deduced from the pairs listed in Table 6 by either exchanging $x$ and $y$ or by multiplying them by the same power of 10 (results for the decimal64 format are listed in Table 9 in the Appendix, and those for the decimal128 format are available at http://prunel. ccsd.cnrs.fr/ensl-00409366/fr/).

The following table gives the number $N_{z}$ of midpoints $z$ in a decade (i.e., with a fixed exponent $e_{z}$ ), with respect to the decimal format considered. The table also gives the number $N$ of pairs of integral significand $(X, Y)$ with $X \geq Y$ that give these midpoints. In decimal64 arithmetic, for instance, the function $(x, y) \mapsto 1 / \sqrt{x^{2}+y^{2}}$ has two midpoints $z_{1}<z_{2}$ in the decade $[1,10)$ : the number of pairs $(X, Y)$ that give $z_{1}$ is 10 , and nine pairs give $z_{2}$.

\begin{tabular}{|c|c|c|c|}
\hline $\begin{array}{c}\text { Format } \\
p\end{array}$ & $\begin{array}{c}\text { decimal32 } \\
7\end{array}$ & $\begin{array}{c}\text { decimal64 } \\
16\end{array}$ & $\begin{array}{c}\text { decimal128 } \\
34\end{array}$ \\
\hline \hline$N_{z}$ & 1 & 2 & 2 \\
\hline$N$ & 4 & $10+9$ & $20+19$ \\
\hline
\end{tabular}

\subsection{Exact Points for Reciprocal 2D Norm}

Theorem 13. Let $x, y \in \mathbb{F}_{\beta, p}$ be such that $(x, y) \neq(0,0)$. Let $X, Y$ denote the integral significands of $x, y$, and let also $z$ denote $1 / \sqrt{x^{2}+y^{2}}$.

- For $\beta=2^{q}\left(q \in \mathbb{N}_{>0}\right)$, the real $z$ is also in $\mathbb{F}_{2^{q}, p}$ if and only if $\{x, y\}=\left\{0, \pm 2^{k}\right\}$ for some $k \in \mathbb{Z}$.

- For $\beta=10$, the number $z$ is in $\mathbb{F}_{10, p}$ if and only if its integral significand $Z$ satisfies $Z=2^{k} \cdot 5^{\ell}$, with $10^{p-1} \leq 2^{k} \cdot 5^{\ell}<10^{p}$ and $k, \ell \in \mathbb{N}$. In this case, one has $2^{8 p-2 k} \cdot 5^{8 p-2 \ell} \in \mathbb{N}$ and $(X, Y)$ must satisfy

$$
\left(X \cdot 10^{m}\right)^{2}+\left(Y \cdot 10^{n}\right)^{2}=2^{8 p-2 k} \cdot 5^{8 p-2 \ell},
$$

where $m, n \in \mathbb{Z}$ such that $X \cdot 10^{m}$ and $Y \cdot 10^{n}$ are in $\mathbb{N}$.

Proof. Without loss of generality, we assume that $1 \leq z<\beta$ and $0 \leq y \leq x$. Reasoning as in the proof of Theorem 12, one may check that necessarily $e_{x} \in\{-2,-1,0\}$. Using as usual the normalized representations of $x, y$, and $z$, from $\left(x^{2}+y^{2}\right) z=1$, we deduce

$$
Z^{2}\left(X^{2} \cdot \beta^{2 e_{x}-2 e_{y}}+Y^{2}\right)=\beta^{4 p-4-2 e_{y}} .
$$

If $\beta=2^{q}$ for some $q \in \mathbb{N}_{>0}$, then (27) implies that $Z=$ $2^{\ell}$ for some $\ell \in \mathbb{Z}$. From (27), we then deduce 


$$
\left(X \cdot 2^{q\left(e_{x}+2\right)}\right)^{2}+\left(Y \cdot 2^{q\left(e_{y}+2\right)}\right)^{2}=2^{4 q p-2 \ell} .
$$

Since $2^{q(p-1)} \leq Z<2^{q p}$, we deduce that $q(p-1) \leq \ell<q p$; hence, $2^{4 q p-2 \ell}$ is in $\mathbb{N}$. Since both $2^{4 q p-2 \ell}$ and $X \cdot 2^{q\left(e_{x}+2\right)}$ are in $\mathbb{N}$, it follows that $\left(Y \cdot 2^{q\left(e_{y}+2\right)}\right)^{2}$ is also in $\mathbb{N}$, and from Lemma 4, we deduce that $Y \cdot 2^{q\left(e_{y}+2\right)} \in \mathbb{N}$. Then, Corollary 2 implies that the only possible decomposition of $2^{4 q p-2 \ell}$ as the sum of two squares is $2^{4 q p-2 \ell}=0^{2}+\left(2^{2 q p-\ell}\right)^{2}$ so that $\{X, Y\}=\left\{0,2^{2 q p-\ell}\right\}$. Conversely, if $\{x, y\}=$ $\left\{0, \pm 2^{k}\right\}$, then $1 / \sqrt{x^{2}+y^{2}}=2^{-k}$ belongs to $\mathbb{F}_{2^{q}, p}$.

Now, let us assume that $\beta=10$. Then, (27) becomes

$$
Z^{2}\left(X^{2} \cdot 10^{2 e_{x}-2 e_{y}}+Y^{2}\right)=10^{4 p-4-2 e_{y}} .
$$

Since $10^{4 p-4-2 e_{y}}$ is a multiple of $Z$, necessarily $Z=2^{k} \cdot 5^{\ell}$ with $k, \ell \in \mathbb{N}$ such that $10^{p-1} \leq 2^{k} \cdot 5^{\ell}<10^{p}$, which implies that $\ell \leq 2 p$ and $k \leq 4 p$. Moreover, from (29) with $Z=2^{k} \cdot 5^{\ell}$, we have

$$
\left(X \cdot 10^{2 p+e_{x}+2}\right)^{2}+\left(Y \cdot 10^{2 p+e_{y}+2}\right)^{2}=2^{8 p-2 k} \cdot 5^{8 p-2 \ell} .
$$

Since $\left(X \cdot 10^{2 p+e_{x}+2}\right)^{2}$ and $2^{8 p-2 k} \cdot 5^{8 p-2 \ell}$ are both in $\mathbb{N}$, then $Y \cdot 10^{2 p+e_{y}+2}$ also belongs to $\mathbb{N}$, which concludes the proof.

In radix $2^{q}$, the pairs $(x, y)$ such that $1 / \sqrt{x^{2}+y^{2}}$ is a midpoint are characterized by Theorem 13. In radix 10 , for each $Z=2^{k} \cdot 5^{\ell}$ with $k, \ell \in \mathbb{N}$ such that $10^{p-1} \leq 2^{k} \cdot 5^{\ell}<10^{p}$, we are reduced to find all decompositions of $2^{8 p-2 k} \cdot 5^{8 p-2 \ell}$ as sums of two squares. This is done as explained in Section 8.1. For each basic decimal format of the IEEE 7542008 standard, the following table gives the number $N_{z}$ of midpoints with a fixed exponent $e_{z}$, together with the number $N$ of pairs of significands $(X, Y)$ with $X \geq Y$ such that $1 / \sqrt{x^{2}+y^{2}}$ is in $\mathbb{F}_{10, p}$.

\begin{tabular}{|c|c|c|c|}
\hline $\begin{array}{c}\text { Format } \\
p\end{array}$ & $\begin{array}{c}\text { decimal32 } \\
7\end{array}$ & $\begin{array}{c}\text { decimal64 } \\
16\end{array}$ & $\begin{array}{c}\text { decimal128 } \\
34\end{array}$ \\
\hline \hline$N_{z}$ & 42 & 93 & 196 \\
\hline$N$ & 160 & 764 & 3373 \\
\hline
\end{tabular}

\section{Normalization of 2D Vectors}

Theorem 7 shows that $x / \sqrt{x^{2}+y^{2}}$ cannot be a midpoint in radix 2 . Here, we first extend this result to radices $2^{q}$ and 10 . Then, we characterize the exact points of the 2Dnormalization function in radix $2^{q}$.

\subsection{Midpoints for 2D Normalization}

Theorem 14. Let $x, y \in \mathbb{F}_{\beta, p}$ such that $(x, y) \neq(0,0)$. If $\beta=2^{q}$ $\left(q \in \mathbb{N}_{>0}\right)$ or $\beta=10$, then $x / \sqrt{x^{2}+y^{2}} \notin \mathbb{M}_{\beta, p}$.

Proof. Without loss of generality, let us assume that $x, y>0$, and assume that $z=x / \sqrt{x^{2}+y^{2}}$ is a midpoint. Hence, we write as usual $z=(Z+1 / 2) \cdot 10^{e_{z}-p+1}$ with $e_{z} \in \mathbb{Z}$ and $Z \in$ $\mathbb{N}$ such that $\beta^{p-1} \leq Z<\beta^{p}$. From $x / \sqrt{x^{2}+y^{2}} \leq 1$, we deduce that $z \leq 1$; hence, $e_{z} \leq 0$. Using $x^{2}\left(1-z^{2}\right)=y^{2} z^{2}$ and the normalized representations of $x$ and $y$ gives

$X^{2}\left(4 \cdot \beta^{2 p-2-2 e_{z}}-(2 Z+1)^{2}\right)=Y^{2}(2 Z+1)^{2} \cdot \beta^{2 e_{y}-2 e_{x}}$.

From $e_{z} \leq 0$, the left-hand side of (31) is in $\mathbb{N}$, and thus, using Lemma $4, Y(2 Z+1) \cdot \beta^{e_{y}-e_{x}} \in \mathbb{N}$. Since $Y^{2}(2 Z+1)^{2} \cdot \beta^{2 e_{y}-2 e_{z}}$ is a multiple of $X^{2}$, it follows that
$Y(2 Z+1) \cdot \beta^{e_{y}-e_{x}}=J X$ for some $J$ in $\mathbb{N}_{>0}$. Equation (31) then becomes

$$
\left(2 \cdot \beta^{p-1-e_{z}}\right)^{2}=J^{2}+(2 Z+1)^{2},
$$

which expresses $\left(2 \cdot \beta^{p-1-e_{z}}\right)^{2}$ as a sum of two integer squares.

If $\beta=2^{q}$, then $\left(2 \cdot \beta^{p-1-e_{z}}\right)^{2}$ is an even power of two, and Corollary 2 then implies that it has only one possible decomposition, which is $0^{2}+\left(2^{q\left(p-1-e_{z}\right)+1}\right)^{2}$. However, this contradicts the fact that both $J$ and $2 Z+1$ are positive integers.

With $\beta=10$, (32) becomes

$$
2^{2 p-2 e_{z}} \cdot 5^{2 p-2-2 e_{z}}=J^{2}+(2 Z+1)^{2} .
$$

Since $2 p-2 e_{z}$ is even, according to Theorem 11, one has

$$
2^{2 p-2 e_{z}} \cdot 5^{2 p-2-2 e_{z}}=|\Re(c)|^{2}+|\Im(c)|^{2},
$$

with $c=2^{p-e_{z}}(2+i)^{k}(2-i)^{2 p-2-2 e_{z}-k}$ for some $k \in \mathbb{N}$, and one may check that both $|\Re(c)|$ and $|\Im(c)|$ are even. Hence, the two squares in the right-hand side of (33) must be even, which is a contradiction since $2 Z+1$ is odd.

\subsection{Exact Points for 2D Normalization}

The next theorem provides a characterization of the exact points of the 2D-normalization function in radix $2^{q}$.

Theorem 15. Let $q \in \mathbb{N}_{>0}$ and let $x, y \in \mathbb{F}_{2^{q}, p}$ be such that $(x, y) \neq(0,0)$. One has $z=x / \sqrt{x^{2}+y^{2}} \in \mathbb{F}_{2^{q}, p}$ if and only if $x=0$ or $y=0$.

Proof. The "if" statement is obvious. Conversely, assume that $z \in \mathbb{F}_{2^{q}, p}$ and both $x$ and $y$ are nonzero. We can restrict to $x, y>0$ with no loss of generality. Let $z=x / \sqrt{x^{2}+y^{2}}$. Since $z \leq 1$, necessarily $e_{z} \leq 0$. Then, using $x^{2}\left(1-z^{2}\right)=y^{2} z^{2}$ and the normalized representations of $x$ and $y$,

$$
X^{2}\left(\beta^{2 p-2 e_{z}-2}-Z^{2}\right)=Y^{2} Z^{2} \cdot \beta^{2 e_{y}-2 e_{x}} .
$$

From $e_{z} \leq 0$, it follows that the left-hand side of (34) is in $\mathbb{N}$ and, due to Lemma 4 , so is $Y Z \cdot \beta^{e_{y}-e_{y}}$. Now, since $Z^{2} Y^{2} \cdot \beta^{2 e_{y}-2 e_{x}}$ is a multiple of $X^{2}$, we have $Z Y \cdot \beta^{e_{y}-e_{x}}=$ $J X$ for some $J \in \mathbb{N}_{>0}$. Then, we obtain from (34)

$$
\left(\beta^{e_{z}-p+1}\right)^{2}=J^{2}+Z^{2} .
$$

When $\beta=2^{q}$, Corollary 2 implies that either $J$ or $Z$ is zero, a contradiction.

In radix 10, we do not have simple results to characterize the exact points of the 2D-normalization function. But they can, of course, be enumerated using (35) at least for some small precisions. Using Theorem 11, we enumerate all the pairs $(Z, J)$ for a fixed $e_{z}$ such that (35) holds. Without loss of generality, we fix $e_{x}=0$. The inputs $x$ and $y$ can then be found by searching the points $\left(X, Y \cdot 10^{e_{y}}\right)$ on the line $Y Z \cdot 10^{e_{y}}=J X$, with $10^{p-1} \leq X<10^{p}$ and $X \in \mathbb{N}$. For some small precisions, the following table gives the number of pairs of inputs $(X, Y)$ such that $x / \sqrt{x^{2}+y^{2}}$ is an exact point.

\begin{tabular}{|c|c|c|c|c|c|c|c|}
\hline$p$ & 1 & 2 & 3 & 4 & 5 & 6 & 7 \\
\hline \hline$e_{z}=-1$ & 4 & 54 & 558 & 5622 & 56254 & 562696 & 5630268 \\
\hline$e_{z}=-2$ & 0 & 0 & 0 & 6 & 60 & 597 & 2889 \\
\hline
\end{tabular}


This experiment suggests that the number of pairs $(x, y)$ such that $x / \sqrt{x^{2}+y^{2}}$ is an exact point grows very rapidly with $p$, and no useful enumeration can be performed.

\section{2D EuClidean Norm}

Let $x$ and $y$ be two numbers in $\mathbb{F}_{\beta, p}$, and assume that $z=$ $\sqrt{x^{2}+y^{2}}$ is a midpoint. We use the normalized representations of $x$ and $y$, and write as usual $z=(Z+1 / 2) \cdot \beta^{e_{z}-p+1}$. Without loss of generality, we assume that $x \geq y$, which implies that $e_{z} \geq e_{x} \geq e_{y}$. Then, from $x^{2}+y^{2}=z^{2}$, it follows that

$$
4\left(Y^{2}+X^{2} \cdot \beta^{2 e_{x}-2 e_{y}}\right)=(2 Z+1)^{2} \cdot \beta^{2 e_{z}-2 e_{y}} .
$$

When $\beta$ is odd, the right-hand side of (36) is odd, while the left-hand side is always even. Hence, if the radix $\beta$ is odd, $\sqrt{x^{2}+y^{2}}$ cannot be a midpoint, and this observation can be generalized to the euclidean norm in higher dimensions. Nevertheless, this not a very useful result since it does not hold for binary, decimal, nor hexadecimal arithmetic.

For even radices, we do not have general results. Equation (36) has solutions, and exhaustive enumeration can be performed at least for small precisions. In radices 2 and 10, and for some small precisions $p$, the following two tables display the number $N$ of input pairs $(x, y)$, with $x \geq y$, such that $z=\sqrt{x^{2}+y^{2}}$ is a midpoint in $[1, \beta)$.

Radix 2
\begin{tabular}{|c|c|c|c|c|c|c|c|c|c|c|}
\hline$p$ & 1 & 2 & 3 & 4 & 5 & 6 & 7 & 8 & 9 & 10 \\
\hline \hline$N$ & 0 & 1 & 1 & 3 & 5 & 18 & 30 & 76 & 155 & 348 \\
\hline
\end{tabular}

Radix 10
\begin{tabular}{|c|c|c|c|c|}
\hline$p$ & 1 & 2 & 3 & 4 \\
\hline \hline$N$ & 0 & 11 & 177 & 2428 \\
\hline
\end{tabular}

These experiments suggest that the number of midpoints for the function $(x, y) \mapsto \sqrt{x^{2}+y^{2}}$ grows very rapidly with $p$.

On the other hand, in one dimension, the euclidean norm reduces to the absolute value, which suffices to see that it admits only exact points.

\section{Conclusion}

We have shown that for several simple algebraic functions $\left(\sqrt{y}, 1 / \sqrt{y}, x^{k}\right.$ for $k \in \mathbb{N}_{>0}, x /\|y\|_{2}, x / y, 1 / y, 1 / \sqrt{x^{2}+y^{2}}$, $\left.x / \sqrt{x^{2}+y^{2}}\right)$, we can obtain useful information on the existence of midpoints and exact points. This information can be used for simplifying or improving the performance of programs that evaluate these functions.

Finding midpoints and exact points would also be of interest for the most common transcendental functions (sine, cosine, exponential, logarithm,...). Providing these functions with correct rounding is a difficult problem, known as the Table-Maker's Dilemma [7], [10]. For the most simple transcendental functions, these built from the complex exponential and logarithm, one can deduce the nonexistence of midpoints from the following corollary of Lindemann's theorem (see, for example, [2, p. 6]):

Theorem 16 (Lindemann). $e^{z}$ is transcendental for every nonzero algebraic complex number $z$.
TABLE 7

List of the 45 Integral Significands $Y$ Such That $1 / y$ Is an Exact Point in the Case of the Decimal64 Format

\begin{tabular}{|c|c|}
\hline$Y$ & $1 / Y$ \\
\hline 2384185791015625 & $4.194304000000000 \cdot 10^{-16}$ \\
\hline 4768371582031250 & $2.097152000000000 \cdot 10^{-16}$ \\
\hline 1907348632812500 & $5.242880000000000 \cdot 10^{-16}$ \\
\hline 9536743164062500 & $1.048576000000000 \cdot 10^{-16}$ \\
\hline 3814697265625000 & $2.621440000000000 \cdot 10^{-16}$ \\
\hline 1525878906250000 & $6.553600000000000 \cdot 10^{-16}$ \\
\hline 7629394531250000 & $1.310720000000000 \cdot 10^{-16}$ \\
\hline 3051757812500000 & $3.276800000000000 \cdot 10^{-16}$ \\
\hline 1220703125000000 & $8.192000000000000 \cdot 10^{-16}$ \\
\hline 6103515625000000 & $1.638400000000000 \cdot 10^{-16}$ \\
\hline 2441406250000000 & $4.096000000000000 \cdot 10^{-16}$ \\
\hline 4882812500000000 & $2.048000000000000 \cdot 10^{-16}$ \\
\hline 1953125000000000 & $5.120000000000000 \cdot 10^{-16}$ \\
\hline 9765625000000000 & $1.024000000000000 \cdot 10^{-16}$ \\
\hline 3906250000000000 & $2.560000000000000 \cdot 10^{-16}$ \\
\hline 1562500000000000 & $6.400000000000000 \cdot 10^{-16}$ \\
\hline 7812500000000000 & $1.280000000000000 \cdot 10^{-16}$ \\
\hline 3125000000000000 & $3.200000000000000 \cdot 10^{-16}$ \\
\hline 1250000000000000 & $8.000000000000000 \cdot 10^{-16}$ \\
\hline 6250000000000000 & $1.600000000000000 \cdot 10^{-16}$ \\
\hline 2500000000000000 & $4.000000000000000 \cdot 10^{-16}$ \\
\hline 1000000000000000 & $1.000000000000000 \cdot 10^{-15}$ \\
\hline 5000000000000000 & $2.000000000000000 \cdot 10^{-16}$ \\
\hline 2000000000000000 & $5.000000000000000 \cdot 10^{-16}$ \\
\hline 4000000000000000 & $2.500000000000000 \cdot 10^{-16}$ \\
\hline 1600000000000000 & $6.250000000000000 \cdot 10^{-16}$ \\
\hline 8000000000000000 & $1.250000000000000 \cdot 10^{-16}$ \\
\hline 3200000000000000 & $3.125000000000000 \cdot 10^{-16}$ \\
\hline 1280000000000000 & $7.812500000000000 \cdot 10^{-16}$ \\
\hline 6400000000000000 & $1.562500000000000 \cdot 10^{-16}$ \\
\hline 2560000000000000 & $3.906250000000000 \cdot 10^{-16}$ \\
\hline 1024000000000000 & $9.765625000000000 \cdot 10^{-16}$ \\
\hline 5120000000000000 & $1.953125000000000 \cdot 10^{-16}$ \\
\hline 2048000000000000 & $4.882812500000000 \cdot 10^{-16}$ \\
\hline 4096000000000000 & $2.441406250000000 \cdot 10^{-16}$ \\
\hline 1638400000000000 & $6.103515625000000 \cdot 10^{-16}$ \\
\hline 8192000000000000 & $1.220703125000000 \cdot 10^{-16}$ \\
\hline 3276800000000000 & $3.051757812500000 \cdot 10^{-16}$ \\
\hline 1310720000000000 & $7.629394531250000 \cdot 10^{-16}$ \\
\hline 6553600000000000 & $1.525878906250000 \cdot 10^{-16}$ \\
\hline 2621440000000000 & $3.814697265625000 \cdot 10^{-16}$ \\
\hline 1048576000000000 & $9.536743164062500 \cdot 10^{-16}$ \\
\hline 5242880000000000 & $1.907348632812500 \cdot 10^{-16}$ \\
\hline 2097152000000000 & $4.768371582031250 \cdot 10^{-16}$ \\
\hline 4194304000000000 & $2.384185791015625 \cdot 10^{-16}$ \\
\hline
\end{tabular}

Since floating-point numbers as well as midpoints are algebraic numbers, Theorem 16 allows us to deduce that for any radix and precision, if $x$ is a floating-point number, then $\ln (x), \exp (x), \sin (x), \cos (x), \tan (x), \arctan (x), \arcsin (x)$, and $\arccos (x)$ cannot be midpoints. Furthermore, the only exact points are $\ln (1)=0, \exp (0)=1, \sin (0)=0, \cos (0)=1$, $\tan (0)=0, \arctan (0)=0, \arcsin (0)=0$, and $\arccos (1)=0$.

The case of radix-2 and radix-10 exponentials and logarithms has to be treated more carefully. But one can prove that the radix-2 or 10 logarithm of a rational number is either an integer or an irrational number. This gives the following result. Assume that the exponent size is less than the precision (which is true in any reasonable floating-point system) and $x$ is a floating-point number. Then, we have the following:

- $\log _{2}(x)$ cannot be a midpoint. It can be an exact point only when $x=2^{k}$, where $k$ is an integer; 
TABLE 8

All Integral Significands $Y$ of $y$, and the Parity of the Exponent $e_{y}$, Such That $z=1 / \sqrt{y}$ Is a Floating-Point Number Too, in Decimal64 Format

\begin{tabular}{|c|c|c|c|}
\hline & $Y$ & $1 / \sqrt{Y \cdot 10^{\delta_{y}-p+1}}$ & $\delta_{y}$ \\
\hline $2^{15} \cdot 5^{15}$ & $=10000000000$ & 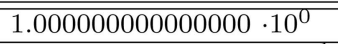 & 0 \\
\hline $2^{45} \cdot 5^{3}$ & $=4398046511104000$ & $4.768371582031250 \cdot 10^{-1}$ & 0 \\
\hline $2^{43} \cdot 5^{3}$ & $=1099511627776000$ & $9.536743164062500 \cdot 10^{-1}$ & 0 \\
\hline $2^{41} \cdot 5^{5}$ & $=6871947673600000$ & $3.814697265625000 \cdot 10^{-1}$ & 0 \\
\hline $2^{39} \cdot 5^{5}$ & $=1717986918400000$ & $7.629394531250000 \cdot 10^{-1}$ & 0 \\
\hline $2^{35} \cdot 5^{7}$ & $=2684354560000000$ & $6.103515625000000 \cdot 10^{-1}$ & 0 \\
\hline $2^{31} \cdot 5^{9}$ & $=4194304000000000$ & $4.882812500000000 \cdot 10^{-1}$ & 0 \\
\hline $2^{29} \cdot 5^{9}$ & $=1048576000000000$ & $9.765625000000000 \cdot 10^{-1}$ & 0 \\
\hline $2^{27} \cdot 5^{11}$ & $=655360$ & $3.906250000000000 \cdot 10^{-1}$ & 0 \\
\hline $2^{25} \cdot 5^{11}$ & 000000000 & $7.812500000000000 \cdot 10^{-1}$ & 0 \\
\hline $2^{21} \cdot 5^{13}$ & $=25600$ & $6.250000000000000 \cdot 10^{-1}$ & 0 \\
\hline $2^{17} \cdot 5^{15}$ & $=40000$ & $5.000000000000000 \cdot 10^{-1}$ & 0 \\
\hline $2^{13} \cdot 5^{17}$ & $=625$ & $4.000000000000000 \cdot 10^{-1}$ & 0 \\
\hline $2^{11} \cdot 5^{17}$ & $=156250$ & $8.000000000000000 \cdot 10^{-1}$ & 0 \\
\hline $2^{9} \cdot 5^{19}$ & $=9765625000000000$ & $3.200000000000000 \cdot 10^{-1}$ & 0 \\
\hline $2^{7} \cdot 5^{19}$ & $=2441406250000000$ & $6.400000000000000 \cdot 10^{-1}$ & 0 \\
\hline $2^{3} \cdot 5^{21}$ & $=38146$ & $5.120000000000000 \cdot 10^{-1}$ & 0 \\
\hline $2^{46} \cdot 5^{2}$ & $=1759218604441600$ & $2.384185791015625 \cdot 10^{-1}$ & 1 \\
\hline $2^{42} \cdot 5^{4}$ & $=2748779069440000$ & $1.907348632812500 \cdot 10^{-1}$ & 1 \\
\hline $2^{38} \cdot 5^{6}$ & $=4294967296000000$ & $1.525878906250000 \cdot 10^{-1}$ & 1 \\
\hline $2^{36} \cdot 5^{6}$ & $=1073741824000000$ & $3.051757812500000 \cdot 10^{-1}$ & 1 \\
\hline $2^{34} \cdot 5^{8}$ & $=6710886400000000$ & $1.220703125000000 \cdot 10^{-1}$ & 1 \\
\hline $2^{32} \cdot 5^{8}$ & $=1677721600000000$ & $2.441406250000000 \cdot 10^{-1}$ & 1 \\
\hline $2^{28} \cdot 5^{10}$ & $=2621440000000000$ & $1.953125000000000 \cdot 10^{-1}$ & 1 \\
\hline $2^{24} \cdot 5^{12}$ & $=4096000000000000$ & $1.562500000000000 \cdot 10^{-1}$ & 1 \\
\hline $2^{22} \cdot 5^{12}$ & $=1024000000000000$ & $3.125000000000000 \cdot 10^{-1}$ & 1 \\
\hline $2^{20} \cdot 5^{14}$ & $=6400000000000000$ & $1.250000000000000 \cdot 10^{-1}$ & 1 \\
\hline $2^{18} \cdot 5^{14}$ & $=1600000000000000$ & $2.500000000000000 \cdot 10^{-1}$ & 1 \\
\hline $2^{14} \cdot 5^{16}$ & $=2500000000000000$ & $2.000000000000000 \cdot 10^{-1}$ & 1 \\
\hline $2^{10} \cdot 5^{18}$ & $=3906250000000000$ & $1.600000000000000 \cdot 10^{-1}$ & 1 \\
\hline $2^{6} \cdot 5^{20}$ & $=6103515625000000$ & $1.280000000000000 \cdot 10^{-1}$ & 1 \\
\hline & $=1525878906250000$ & $2.560000000000000 \cdot 10^{-1}$ & 1 \\
\hline $2^{2} \cdot 5^{22}$ & $=9536743164062500$ & $1.024000000000000 \cdot 10^{-1}$ & 1 \\
\hline $2^{0} \cdot 5^{22}$ & $=2384185791015625$ & $2.048000000000000 \cdot 10^{-}$ & 1 \\
\hline
\end{tabular}

- $\log _{10}(x)$ cannot be a midpoint. It can be an exact point only when $x=10^{k}$, where $k$ is an integer.

It is always possible to build ad hoc transcendental functions for which something can be said about midpoints or exact points. Unfortunately, for the many common nonelementary transcendental functions useful in scientific applications (physics, statistics, etc.), almost nothing is known about their midpoints or exact points in floatingpoint arithmetic.

Consider, for instance, the Gamma function. We know that if $n$ is a nonnegative integer, then $\Gamma(n)=(n-1)$ ! is an integer too (which implies the existence of midpoints in some cases, e.g., in radix-2 arithmetic with $p=3$, the number $6_{10}=110_{2}$ is a floating-point number, and $\Gamma(6)=$ $5 !=120_{10}=1111000_{2}$ is a midpoint). Although we have no proof of that, it is extremely unlikely that Gamma of a noninteger floating-point number could be a midpoint or an exact point. To our knowledge (see, for example, [14]), the only result that can be used to deal with a very few cases is that $\Gamma(x)$ is shown to be irrational if $x$ modulo 1 belongs to $\{1 / 6,1 / 4,1 / 3,1 / 2,2 / 3,3 / 4,5 / 6\}$.

\section{APPENDIX}

Exact Points for Reciprocal, Square Root RECIPROCAL, AND 2D-NORM RECIPROCAL
TABLE 9

In Decimal64 Arithmetic, the Function $1 / \sqrt{x^{2}+y^{2}}$ Has Two Midpoints in the Range $\left[10^{-16}, 10^{-15}\right)$, Denoted by $z_{1}$ and $z_{2}$ : The Pairs of Floating-Point Numbers $x$ and $y$ Such That $1 / \sqrt{x^{2}+y^{2}}$ Equals $z_{1}$ or $z_{2}$ Are Listed Below

\begin{tabular}{|c|c|c|}
\hline$x$ & $y$ & $z$ \\
\hline 8053063680000000 & 2348810240000000 & $z_{1}$ \\
\hline 6710886400000000 & 5033164800000000 & $=$ \\
\hline 7851737088000000 & 2952790016000000 & 1.1920928955078125 \\
\hline 7073274265600000 & 4509715660800000 & $\times 10^{-16}$ \\
\hline 6309843828736000 & 5527622909952000 & \\
\hline 8208004625203200 & 1731301317017600 & \\
\hline 7605184490373120 & 3539761721507840 & \\
\hline 7394920071430144 & 3960290559393792 & \\
\hline 8364448808960000 & 636192030720000 & \\
\hline 8388608000000000 & 0 & \\
\hline 1342177280000000 & 1006632960000000 & $z_{2}$ \\
\hline 1261968765747200 & 1105524581990400 & $=$ \\
\hline 1610612736000000 & 469762048000000 & 5.9604644775390625 \\
\hline 1570347417600000 & 590558003200000 & $\times 10^{-16}$ \\
\hline 1414654853120000 & 901943132160000 & \\
\hline 1672889761792000 & 127238406144000 & \\
\hline 1641600925040640 & 346260263403520 & \\
\hline 1521036898074624 & 707952344301568 & \\
\hline 1677721600000000 & 0 & \\
\hline
\end{tabular}

\section{REFERENCES}

[1] American National Standards Institute and Institute of Electrical and Electronic Engineers, IEEE Standard for Binary Floating-Point Arithmetic, ANSI/IEEE Standard 754-1985, 1985.

[2] A. Baker, Transcendental Number Theory. Cambridge Univ. Press, 1975.

[3] G. Everest and T. Ward, "An Introduction to Number Theory," Graduate Texts in Mathematics, Springer-Verlag, 2005.

[4] R.L. Graham, D.E. Knuth, and O. Patashnik, Concrete Mathematics, second ed. Addison-Wesley, 1994.

[5] IEEE Computer Society, IEEE Standard for Floating-Point Arithmetic, IEEE Standard 754-2008, http://ieeexplore.ieee.org/ servlet/opac?punumber $=4610933$, Aug. 2008.

[6] C. Iordache and D.W. Matula, "On Infinitely Precise Rounding for Division, Square Root, Reciprocal and Square Root Reciprocal," Proc. 14th IEEE Symp. Computer Arithmetic, I. Koren and P. Kornerup, eds., pp. 233-240, Apr. 1999.

[7] W. Kahan, "A Logarithm Too Clever by Half," http:// http.cs.berkeley.edu/ wkahan/LOG10HAF.TXT, 2004.

[8] C.Q. Lauter and V. Lefèvre, "An Efficient Rounding Boundary Test for $\operatorname{Pow}(x, y)$ in Double Precision," IEEE Trans. Computers, vol. 58, no. 2, pp. 197-207, Feb. 2009.

[9] P. Markstein, IA-64 and Elementary Functions: Speed and Precision Prentice-Hall, 2000.

[10] J.-M. Muller, Elementary Functions, Algorithms and Implementation, second ed. Birkhäuser, 2006.

[11] M. Parks, "Inexact Quotients and Square Roots," http:// www.geocities.com/ieee754/papers/parks-inexact.ps, 2010.

[12] E.M. Schwarz, R.M. Smith, and C.A. Krygowski, "The S/390 G5 Floating-Point Unit Supporting Hex and Binary Architectures," Proc. 14th IEEE Symp. Computer Arithmetic (ARITH-14), pp. 258265, Apr. 1999.

[13] S. Wagon, "The Euclidean Algorithm Strikes Again," The Am. Math. Monthly, vol. 97, no. 2, pp. 125-129, Feb. 1990.

[14] M. Waldschmidt, "Transcendence of Periods: The State of the Art," Pure and Applied Math. Quarterly, vol. 2, no. 2, pp. 435-463, 2006.

[15] A. Ziv, "Fast Evaluation of Elementary Mathematical Functions with Correctly Rounded Last Bit," ACM Trans. Math. Software, vol. 17 , no. 3, pp. 410-423, Sept. 1991.

See Tables 7, 8, and 9. 


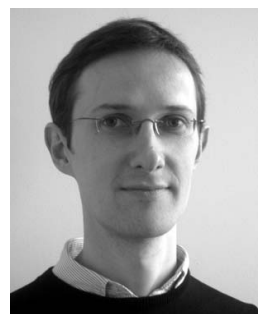

Claude-Pierre Jeannerod received the $\mathrm{PhD}$ degree in applied mathematics from the Institut National Polytechnique de Grenoble in 2000. After being a postdoctoral fellow in the Symbolic Computation Group at the University of Waterloo, he is now a researcher at INRIA GrenobleRhône-Alpes, and a member of the LIP Laboratory (LIP is a joint laboratory of CNRS, École Normale Supérieure de Lyon, INRIA, and Université Claude Bernard Lyon 1). His research interests include computer algebra, linear algebra, and floating-point arithmetic. He is a member of the ACM and the IEEE.

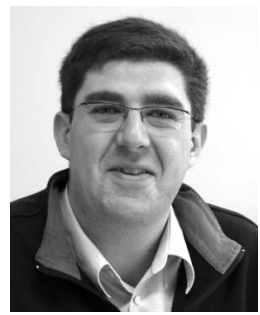

Nicolas Louvet received the MSc degree from the Université de Picardie Jules Verne, Amiens, France, in 2004, and the PhD degree in computer science from the Université de Perpignan Via Domitia, France, in 2007. After being an INRIA postdoctoral fellow in the Arénaire research team, he is now an assistant professor in the Department of Computer Science of the Université Claude Bernard Lyon 1, France, and a member of the LIP Laboratory (LIP is a joint laboratory of CNRS, École Normale Supérieure de Lyon, INRIA, and Université Claude Bernard Lyon 1). His research interests are in computer arithmetic.

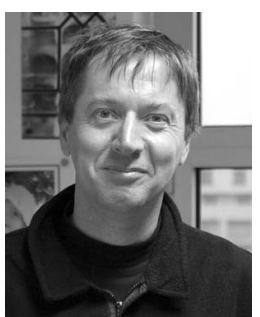

Jean-Michel Muller received the PhD degree from the Institut National Polytechnique de Grenoble in 1985. He is directeur de Recherches (senior researcher) at CNRS, France, and the former head of the LIP laboratory (LIP is a joint laboratory of CNRS, École Normale Supérieure de Lyon, INRIA, and Université Claude Bernard Lyon 1). His research interests are in Computer Arithmetic. He was the coprogram chair of the 13th IEEE Symposium on Computer Arithmetic (Asilomar, June 1997), the general chair of SCAN '97 (Lyon, France, September 1997), and the general chair of the 14th IEEE Symposium on Computer Arithmetic (Adelaide, Australia, April 1999). He is the author of several books, including Elementary Functions, Algorithms and Implementation (second edition, Birkhäuser, Boston, 2006), and coordinated the writing of the Handbook of FloatingPoint Arithmetic (Birkhäuser, Boston, 2010). He served as an associate editor of the IEEE Transactions on Computers from 1996 to 2000 . He is a senior member of the IEEE.

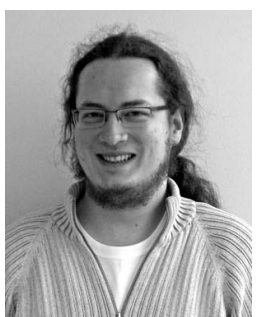

Adrien Panhaleux received the master's degree in 2008 from the École Normale Supérieure de Lyon, where he is currently working toward the PhD degree, under the supervision of JeanMichel Muller and Nicolas Louvet.

$\triangleright$ For more information on this or any other computing topic, please visit our Digital Library at www.computer.org/publications/dlib. 